\title{
Revealing Efficient Dust Formation at Low Metallicity in Extragalactic Carbon-rich Wolf-Rayet Binaries
}

\author{
Ryan M. Lau ${ }^{1}$, Matthew J. Hankins ${ }^{2,3}$ (10, Mansi M. Kasliwal ${ }^{3}$, , Howard E. Bond ${ }^{4,5}$ (), Kishalay De ${ }^{3}$, Jacob E. Jencson ${ }^{3}$, \\ Anthony F. J. Moffat ${ }^{6,7}$, Nathan Smith $^{8}$ (iD, and Peredur M. Williams ${ }^{9}$ (iD) \\ ${ }^{1}$ Institute of Space \& Astronautical Science, Japan Aerospace Exploration Agency, 3-1-1 Yoshinodai, Chuo-ku, Sagamihara, Kanagawa 252-5210, Japan \\ ryanlau@ir.isas.jaxa.jp \\ 2 Department of Physical Sciences, Arkansas Tech University, 1701 N. Boulder Avenue, Russellville, AR 72801, USA \\ ${ }^{3}$ Division of Physics, Mathematics, and Astronomy, California Institute of Technology, Pasadena, CA 91125, USA \\ ${ }^{4}$ Department of Astronomy \& Astrophysics, Pennsylvania State University, University Park, PA 16802, USA \\ ${ }^{5}$ Space Telescope Science Institute, 3700 San Martin Drive, Baltimore, MD 21218, USA \\ ${ }^{6}$ Département de physique, Université de Montréal, C.P. 6128, succ. center-ville, Montréal (Qc) H3C 3J7, Canada \\ ${ }^{7}$ Centre de Recherche en Astrophysique du Québec, Canada \\ ${ }^{8}$ University of Arizona, Steward Observatory, 933 N. Cherry Avenue, Tucson, AZ 85721, USA \\ ${ }^{9}$ Institute for Astronomy, University of Edinburgh, Royal Observatory, Edinburgh EH9 3HJ, UK \\ Received 2020 November 13; revised 2021 January 4; accepted 2021 January 4; published 2021 March 10
}

\begin{abstract}
We present Spitzer/InfraRed Array Camera observations of dust formation from six extragalactic carbon-rich Wolf-Rayet (WC) binary candidates in low-metallicity $\left(Z \lesssim 0.65 Z_{\odot}\right)$ environments using multiepoch mid-infrared (IR) imaging data from the SPitzer InfraRed Intensive Transients Survey (SPIRITS). Optical follow-up spectroscopy of SPIRITS 16ln, 19q, 16df, 18hb, and 14apu reveals emission features from C IV $\lambda 5801-12$ and/or the C III-IV $\lambda 4650 / \mathrm{He}$ II $\lambda 4686$ blend that are consistent with early-type WC stars. We identify SPIRITS 16ln as the variable mid-IR counterpart of the recently discovered colliding-wind WC4 + O binary candidate, N604WRXc, located in the subsolar metallicity NGC $604 \mathrm{H}$ II region in M33. We interpret the mid-IR variability from SPIRITS $161 \mathrm{n}$ as a dust-formation episode in an eccentric colliding-wind WC binary. SPIRITS 19q, 16df, 14apu, and 18hb exhibit absolute [3.6] magnitudes exceeding that of one of the most IR-luminous dust-forming WC systems known, WR $104\left(M_{[3.6]} \lesssim-12.3\right)$. An analysis of dust formation in the mid-IR outburst from SPIRITS 19q reveals a high dust production rate of $\dot{M}_{d} \gtrsim 2 \times 10^{-6} M_{\odot} \mathrm{yr}^{-1}$, which may therefore exceed that of the most efficient dust-forming WC systems known. We demonstrate that efficient dust formation is feasible from early-type WC binaries in the theoretical framework of colliding-wind binary dust formation if the systems host an O-type companion with high mass-loss rates $\left(\dot{M} \gtrsim 1.6 \times 10^{-6} M_{\odot} \mathrm{yr}^{-1}\right)$. This efficient dust formation from early-type WC binaries highlights their potential role as significant sources of dust in low-metallicity environments.
\end{abstract}

Unified Astronomy Thesaurus concepts: Wolf-Rayet stars (1806); WC stars (1793); Circumstellar dust (236); Time domain astronomy (2109); Infrared astronomy (786); Stellar winds (1636)

\section{Introduction}

A classical Wolf-Rayet (WR) star is thought to be the He-rich descendant of a massive O-type star. WR stars are characterized by fast stellar winds $\left(\gtrsim 1000 \mathrm{~km} \mathrm{~s}^{-1}\right)$, hot temperatures $\left(T_{*} \gtrsim 40,000 \mathrm{~K}\right)$, and high luminosities $\left(L_{*} \gtrsim 10^{5}\right.$ $L_{\odot}$; Crowther 2007). Despite the extreme conditions around WR stars that seem inhospitable for dust, there is a subset of carbon-rich WR (WC) stars that exhibits active dust formation (Allen et al. 1972; Gehrz \& Hackwell 1974; Williams et al. 1987). The earliest recorded dust-formation episode around a WC star was based on observations of infrared (IR) variability from the WC7+O5 system WR 140 (as HD 193793; Williams et al. 1978; Hackwell et al. 1979). Dust formation in WC stars is facilitated by colliding-wind interactions between WC star and an OB-star companion (Usov 1991). Dust formation from $\mathrm{WC}+\mathrm{OB}$ binaries is therefore regulated by the orbital properties of the binary system. The link between the orbital configuration and dust formation has been directly observed in several WC+OB-star binaries. One of the most iconic WC binary dust-makers is WR 140, which is a highly eccentric system that exhibits a dust-formation episode every $7.9 \mathrm{yr}$ orbital period when the stars are at their closest orbital separation (Williams et al. 1990; Usov 1991; Williams et al. 2009a). Dust formation has been observed from WC binaries that exhibit orbital periods ranging from 0.7 to $\sim 100 \mathrm{yr}$ (Tuthill et al. 2008; Williams 2019; Han et al. 2020).

Given their capability to output dust at a high rate $\left(\dot{M}_{d} \sim 10^{-10}-10^{-6} M_{\odot} \mathrm{yr}^{-1}\right.$; Zubko 1998; Lau et al. 2020a) and the short timescales associated with the evolutionary onset of the WR phase $(\lesssim \mathrm{Myr})$, WC binaries present a potentially significant and early source of dust. Importantly, WC stars with a binary companion should be common based on the results from the recent spectroscopic survey by Dsilva et al. (2020), who predict that the intrinsic multiplicity fraction of the Galactic WC population is at least $70 \%$.

Recently, Lau et al. (2020a) revisited the impact of dust production from WC binaries on the interstellar medium (ISM) of galaxies in different metallicity environments representative of different epochs in cosmic time. They incorporated dust production into binary population and spectral synthesis (BPASS; Eldridge et al. 2017) models and compared the dust input rates from WC binaries, asymptotic giant branch stars (AGBs), red supergiants (RSGs), and core-collapse supernova ( $\mathrm{SNe}$ ) at different metallicities. SNe were shown to be likely net destroyers of dust, which reaffirmed results from previous studies (e.g., Temim et al. 2015); however, the efficiency of dust destruction from $\mathrm{SN}$ shocks is still disputed (e.g., 
Martínez-González et al. 2019). Lau et al. (2020a) identified the population of WC binaries as significant sources of dust with total dust production rates comparable to the population of AGB stars in solar and Large Magellanic Cloud (LMC)-like $\left(\sim 0.5 Z_{\odot}\right)$ metallicities with constant star formation histories. However, they acknowledged the dearth of known efficient dust-forming WC binaries in the LMC or subsolar environments, which precludes the ability to test model predictions at subsolar metallicities. This issue is exacerbated by the fact that most of the known dust-forming WC binaries are located within the solar circle of our Galaxy (e.g., Rosslowe \& Crowther 2015; Williams 2019), indicating a bias toward studying WC systems at solar or supersolar metallicities.

There is indeed a notable trend in the spectral subtype of WC stars at different metallicities: the cooler, late-type WC stars (WC7-9) are found in high-metallicity environments, whereas the hotter, early-type WC stars (WC4-5) are found at lower metallicities (Massey \& Johnson 1998; Crowther 2007; Hadfield \& Crowther 2007). The early-type WC stars exhibit faster wind velocities $\left(v_{\infty} \sim 3000 \mathrm{~km} \mathrm{~s}^{-1}\right)$ and hotter temperatures $\left(T_{*} \sim 80,000 \mathrm{~K}\right)$ than their late-type counterparts (Sander et al. 2019), which likely impacts the dust-formation efficiency from the colliding-wind mechanism (Usov 1991). Notably, only a few of the 84 known dust-forming WC systems in the Galaxy host early-type WC stars according to the Galactic Wolf Rayet Calalogue V 1.25 (Rosslowe \& Crowther 2015). ${ }^{10}$

Prior to the work presented in this paper, there were only two known dust-forming WC systems beyond our Galaxy. Both of these systems host early-type WC4 stars and are located in the LMC (Bartzakos et al. 2001; Williams et al. 2013; Williams 2019). One of these systems, HD 36402 (=BAT9938), exhibits correlated mid-IR and X-ray variability, which is a strong indicator of dust formation via colliding winds (Williams et al. 2013). Spectroscopic observations of HD 36402 indicate that it is a triple system consistent with a $\mathrm{WC} 4(+\mathrm{O}$ ?) + O8I spectral type, where the inner binary WC4 + O? has a 3.03-day orbital period (Moffat et al. 1990). Multiepoch mid-IR observations revealed variations in dust formation from HD 36402 with a $5.1 \mathrm{yr}$ period, which is presumably associated with the outer orbit with the more distant O8 supergiant (Williams et al. 2013; Williams 2019). The only other extragalactic dust-forming WC system, HD 38030 (=BAT99-84), was recently identified from a midIR brightening event in 2018 (Williams 2019). The dearth of information on extragalactic and early-type dust-forming WC binaries motivates our search to discover and characterize more of these systems.

Dust formation from extragalactic WC binaries is difficult to identify due to the limitations on spatial resolution and the sensitivity of mid-IR observations. Williams (2019) however demonstrated the utility of a multiepoch search for mid-IR variability from colliding-wind WC binaries in our Galaxy and the LMC using $5 \mathrm{yr}$ of archival data from the Near-Earth Object Wide-field Infrared Survey Explorer Reactivation (NEOWISE-R; Mainzer et al. 2014) mission. We are able to drastically expand the search for dust formation from extragalactic WC binaries by using the higher spatial resolution, deeper sensitivity, and $16 \mathrm{yr}$ lifetime of the Spitzer Space Telescope.

\footnotetext{
${ }^{10} \mathrm{http}: /$ pacrowther.staff.shef.ac.uk/WRcat/
}

In this paper, we present mid-IR light curves of six extragalactic dust-forming WC candidates identified from Spitzer/InfraRed Array Camera (IRAC) 3.6 and $4.5 \mu \mathrm{m}$ imaging observations in the SPitzer InfraRed Intensive Transients Survey (SPIRITS; Kasliwal et al. 2017; Karambelkar et al. 2019). One of these candidates includes the recently discovered $\mathrm{WC}+\mathrm{O}$ colliding-wind binary candidate in M33, N604-WRXc (Garofali et al. 2019). For five of the six candidates, we present follow-up optical spectroscopy that reveals emission features consistent with early-type WC stars. We perform a mid-IR color-magnitude diagram (CMD) analysis to determine if the colors exhibited by the candidates are consistent with dust formation and also compare them to other dust-forming and non-dust-forming WC stars. Last, we derive the dust-formation properties for the brightest mid-IR source in our sample, SPIRITS 19q, and discuss the implications and feasibility of dust formation in early-type WC binaries based on the theoretical framework of colliding-wind dust formation presented by Usov (1991).

\section{Observations}

\subsection{Mid-IR Photometry from SPIRITS: The SPitzer InfraRed Intensive Transients Survey}

Mid-IR variability from extragalactic dust-forming WC candidates was identified from the imaging data taken in the SPIRITS survey (Kasliwal et al. 2017). SPIRITS was a systematic, targeted survey of 194 nearby galaxies within $20 \mathrm{Mpc}$ using Channels $1(3.6 \mu \mathrm{m})$ and $2(4.5 \mu \mathrm{m})$ of the IRAC (Fazio et al. 2004) on the Spitzer Space Telescope (Werner et al. 2004; Gehrz et al. 2007) during its Warm Mission. SPIRITS ran from 2014 until the decommissioning of Spitzer in 2020 January. Observations in SPIRITS were performed with cadence baselines ranging from one week to six months and achieved a $5 \sigma$ depth of 20 mag at $3.6 \mu \mathrm{m}$ and $19.1 \mathrm{mag}$ at $4.5 \mu \mathrm{m}$ in the Vega system. All magnitudes provided in this work are given in the Vega units.

The six dust-forming WC candidates SPIRITS 14apu, 14bqe, $16 \mathrm{df}, 16 \mathrm{ln}, 18 \mathrm{hb}$, and 19q were identified from their eruptive or irregular variability and possible associations with $\mathrm{H}$ II regions. These candidates have also been vetted through the SPIRITS image subtraction pipeline (see Kasliwal et al. 2017), which also included archival Spitzer Post Basic Calibrated (PBCD) imaging data from the Spitzer Heritage Archive. ${ }^{11}$ The coordinates and host galaxies of the six SPIRITS sources are summarized in Table 1.

Spitzer/IRAC $25^{\prime \prime} \times 25^{\prime \prime}$ image cutouts and the locations of SPIRITS 16ln, 16df, 19q, 14apu, and 18hb within their host galaxies are shown in Figure 1. The Spitzer/IRAC cutouts for SPIRITS 14bqe are shown in Figure 2. SPIRITS 16ln is located in the H II region NGC 604 on the outskirts of M33. SPIRITS 16df and SPIRITS $19 q$ are both located in bright, dusty $\mathrm{H}$ II regions in NGC 2403. SPIRITS 14apu and SPIRITS $18 \mathrm{hb}$ are located within the dusty spiral arms in the outer regions of M101 and NGC 6946, respectively. Unlike the other five candidates with spiral host galaxies, SPIRITS 14bqe is located in the nearby irregular dwarf galaxy IC 1613.

Photometry was obtained using an aperture with a 4.0 PBCD mosaic pixel (2!"4) radius centered on the coordinates of the sources (Figures 1 and 2). For SPIRITS 14bqe, 16df, 19q,

\footnotetext{
${ }^{11}$ https://sha.ipac.caltech.edu/applications/Spitzer/SHA/
} 
Table 1

SPIRITS Dust-forming WC Candidates

\begin{tabular}{|c|c|c|c|c|c|}
\hline SPIRITS ID & J2000 Coordinates & Galaxy & Distance $(\mu)$ & Distance Ref. & Follow-up Obs. \\
\hline 14bqe & 01:05:01.95 +02:08:51.3 & IC 1613 & $724 \mathrm{kpc}(24.30)$ & Ha17 & WIRC (Imaging) \\
\hline $16 \ln$ & $01: 34: 32.61+30: 47: 04.3$ & Messier 33 & $840 \mathrm{kpc}(24.63)$ & Gi13 & LRS2 \\
\hline $19 q$ & $07: 37: 18.21+65: 33: 49.0$ & NGC 2403 & $3.2 \mathrm{Mpc}(27.51)$ & Ra11 & LRIS, NIRES, LRS2 \\
\hline $16 \mathrm{df}$ & $07: 36: 58.08+65: 37: 23.9$ & NGC 2403 & $3.2 \mathrm{Mpc}(27.51)$ & Ra11 & LRIS \\
\hline 14apu & $14: 03: 31.77+54: 22: 25.1$ & Messier 101 & 6.4 Mpc (29.04) & Sh11 & LRIS \\
\hline $18 \mathrm{hb}$ & $20: 35: 07.68+60: 11: 16.2$ & NGC 6946 & 7.72 Mpc (29.44) & An18 & LRS2 \\
\hline
\end{tabular}

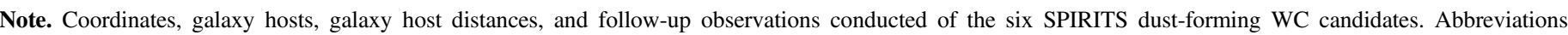

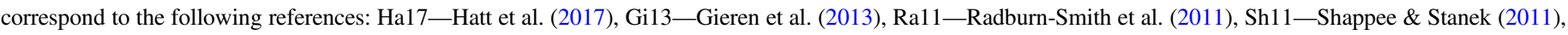
and An18-Anand et al. (2018).
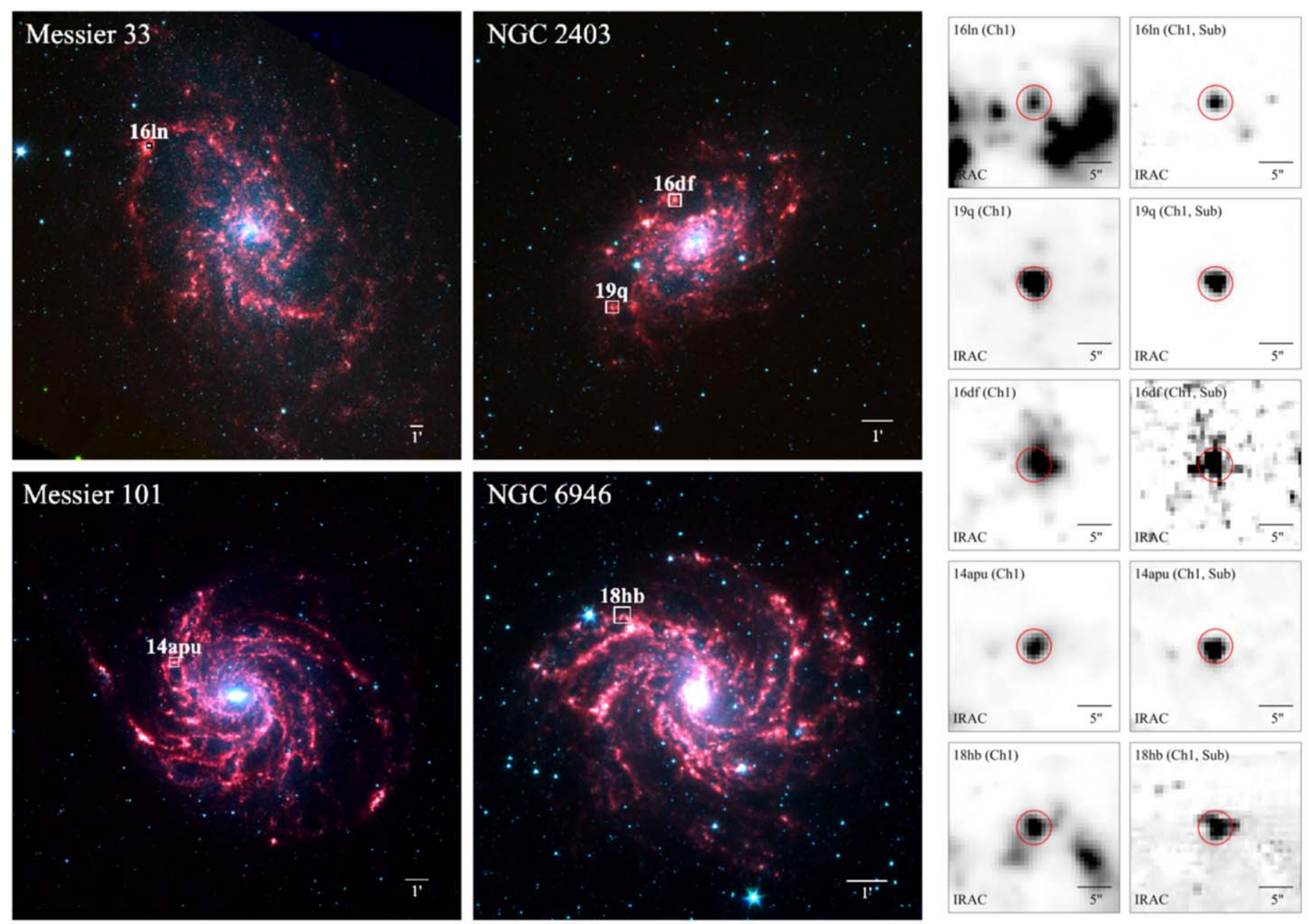

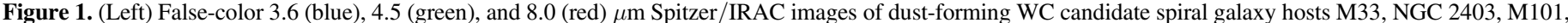

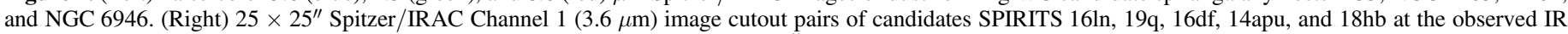

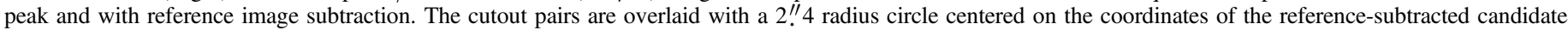
point-spread function.

14apu, and $18 \mathrm{hb}$, a sky background annulus extending from 4.0 to 12.0 mosaic pixels (2!"4-7!'2) was used to subtract the diffuse galaxy background emission. Given the bright emission and crowded region around SPIRITS 16ln, a 24.0-40.0 mosaic pixel (40!"0-66!"7) sky annulus was used to subtract the background emission. The $\mathrm{Ch} 1$ and $\mathrm{Ch} 2$ aperture correction factors for SPIRITS 16ln photometry were 1.208 and 1.220, respectively. For the other five candidates with the 4.0-12.0 mosaic pixel background sky annuli, aperture correction factors of 1.215 and 1.233 were adopted for the Ch1 and $\mathrm{Ch} 2$ photometry, respectively. These aperture-correction factors are consistent with the values provided in the Spitzer/ IRAC manual for the adopted aperture radii and background annuli. Spitzer/IRAC Ch1 and Ch2 photometry and observation dates of all six dust-forming WC candidates are provided in Table 2 . 

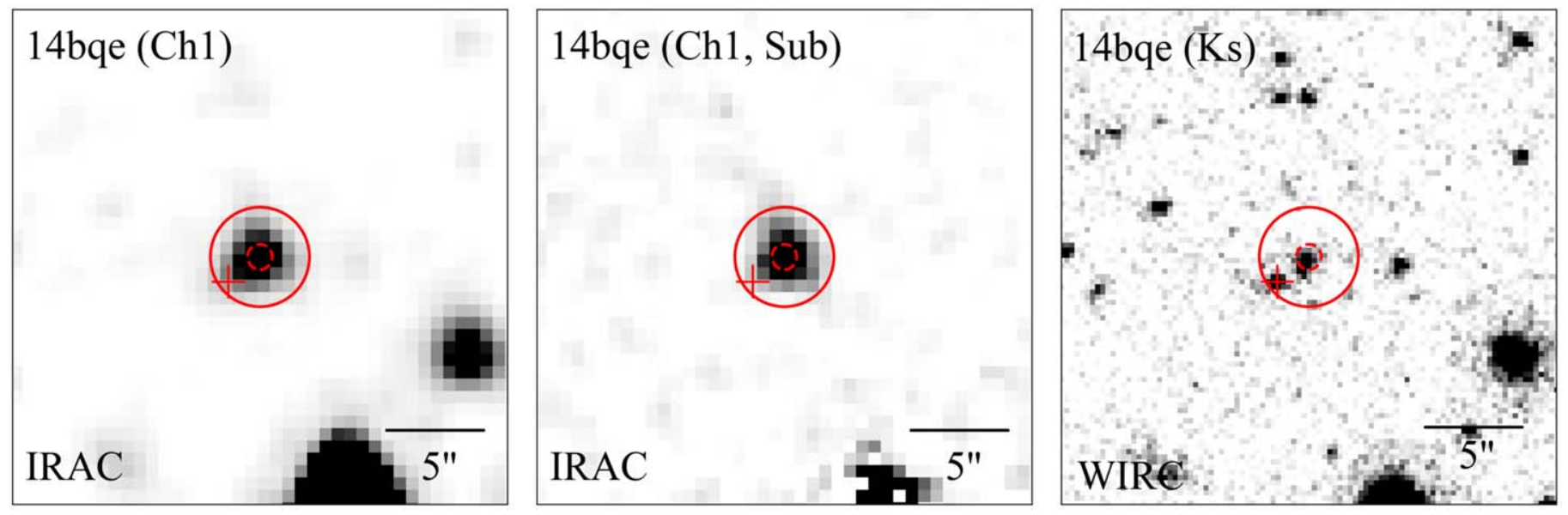

Figure 2. (Left) Spitzer/IRAC Ch1 and (center) reference-subtracted images of SPIRITS 14bqe at IR peak. (Right) WIRC $K_{s}$-band, image of SPIRITS $14 \mathrm{bqe}$ in IC 1613. The images cover the same $25 \times 25^{\prime \prime}$ field of view and are overlaid with two circles centered on SPIRITS 14bqe corresponding to the $2 \sigma(0$ ". 6 ) positional uncertainty (dashed) and the 2 !. 4 radius aperture used for photometry (solid). The plus corresponds to the position of the near-IR southeast neighbor of SPIRITS 14bqe. North is up and east is to the left in all images.

\subsection{Optical Follow-up Spectroscopy}

\subsubsection{Keck I/LRIS}

Optical follow-up spectroscopy of SPIRITS 19q, 16df, and 14apu were obtained using the Low Resolution Imaging Spectrometer (LRIS; Goodrich \& Cohen 2003) on the Keck I telescope. LRIS observations of SPIRITS 19q were conducted on 2019 April 3, and the observations of SPIRITS 16df and 14apu were both taken on 2020 March 23. All three observations used the D560 dichroic with the standard 1". $0 \times 175^{\prime \prime}$ longslit slitmask and the 400/3400 grism on the blue side and the $400 / 8500$ grating on the red side. The resulting spectral resolving power on the blue $(\lambda=3500-6000 \AA)$ and red $(\lambda=5500-10300 \AA)$ sides were $R \sim 600$ and $R \sim 1000$, respectively.

The total exposure times averaged between the red and blue sides for SPIRITS 19q, 16df, and 14apu were $580 \mathrm{~s}, 750 \mathrm{~s}$, and $300 \mathrm{~s}$ respectively. Data were reduced using the fully automated Keck/LRIS reduction software reduction package LPipe (Perley 2019). The flux calibration for SPIRITS 19q was performed using the standard star G191-B2B, and the flux calibrations for SPIRITS 16df and SPIRITS 14apu were performed using Feige34. The reduced and flux-calibrated LRIS spectra of SPIRITS 19q, 16df, and 14apu are shown in Figure 3 . The spectra reveal broad features corresponding to the C IV $\lambda 5801-12$ and/or the C III $\lambda 4650 / \mathrm{C}$ IV $\lambda 4658 / \mathrm{He}$ II $\lambda 4686$ blend as well as a series of narrow emission lines likely originating from underlying or nearby $\mathrm{H}$ II regions. Hereafter, we refer to the combined C III $\lambda 4650 / \mathrm{C}$ IV $\lambda 4658$ components as C III-IV $\lambda 4650$.

\subsubsection{Hobby-Eberly Telescope/LRS2}

Optical follow-up spectroscopy was also conducted with the Low-Resolution Spectrograph 2 (LRS2; Chonis et al. 2016), an integral field spectrograph with a field of view of $6^{\prime \prime} \times 12^{\prime \prime}$ on the $10 \mathrm{~m}$ Hobby-Eberly Telescope (HET). Observations of SPIRITS 16ln, 18hb and 19q were obtained with the blue spectrograph of LRS2, LRS2-B, which covers $3700-4700 \AA(R \approx 1900)$ and $4600-7000 \AA(R \approx 1100)$ in its UV Arm and Orange Arm, respectively. The observations centered on SPIRITS 16ln, 18hb and 19q were taken on 2019
September 8, 2019 August 20, and 2019 October 29, respectively. The total exposure times for SPIRITS $16 \mathrm{ln}, 18 \mathrm{hb}$ and $19 \mathrm{q}$ were $750 \mathrm{~s}, 3 \times 1400 \mathrm{~s}$, and $2 \times 750 \mathrm{~s}$, respectively.

The spectra from the UV and Orange channels were reduced separately using the LRS2 pipeline Panacea. ${ }^{12}$ Since observations were obtained in nonphotometric conditions, the analysis of the LRS2 spectra of SPIRITS 16ln, 18hb, and 19q focuses on the relative flux rather than the absolute flux. The spectra were normalized to the peak emission of the C III-IV $\lambda 4650$ / He II $\lambda 4686$ emission complex in the Orange channel. Due to normalization offsets between the UV and Orange channels, the UV channel emission is offset to match the slope of the continuum emission in the Orange channel. The reduced and normalized LRS2 spectra of SPIRITS 16ln, 18hb, and 19q are shown in Figure 4. Similar to the Keck I/LRIS spectra, the LRS2 spectra show broad features corresponding to the C IV $\lambda 5801-12$ and/or the C III-IV $\lambda 4650 /$ He II $\lambda 4686$ blend as well as a series of narrow emission lines that likely originate from underlying or nearby $\mathrm{H}$ II regions.

\subsection{Near-IR Spectroscopy with Keck II/NIRES}

We obtained a near-IR spectrum of SPIRITS $19 \mathrm{q}$ with the Near-Infrared Echellette Spectrometer ${ }^{13}$ (NIRES) on the Keck II Telescope on 2019 March 13. NIRES uses a 0".55 slit and provides wavelength coverage from 9500 to $24600 \AA$ across five spectral orders with a mean spectral resolving power of $R=2700$. During the observations, we obtained eight individual $300 \mathrm{~s}$ exposures for a total integration time of $2400 \mathrm{~s}$. The target was nodded along the slit between exposures in a standard ABBA pattern to allow for accurate subtraction of the sky background. Observations of the A0V telluric standard star HIP 32549 near the target position were also taken immediately preceding the science target observation for flux calibration and correction of the strong near-IR telluric absorption features. The data were reduced, including flat-fielding, wavelength calibration, background subtraction, and 1D spectral extractions steps, using a version of the IDL-based data reduction

\footnotetext{
12 https://github.com/grzeimann/Panacea

13 https://www2.keck.hawaii.edu/inst/nires/
} 
Table 2

Spitzer/IRAC Photometry of SPIRITS Dust-forming WC Candidates

\begin{tabular}{|c|c|c|c|c|c|c|c|c|}
\hline MJD & $F_{3.6}(\mathrm{mJy})$ & $F_{4.5}(\mathrm{mJy})$ & {$[3.6]$} & [4.5] & {$[3.6]-[4.5]$} & $M_{[3.6]}$ & $M_{[4.5]}$ & PI (Prog ID) \\
\hline \multicolumn{9}{|c|}{ SPIRITS16ln } \\
\hline 53001.39 & $0.632 \pm 0.030$ & $0.600 \pm 0.026$ & $14.12 \pm 0.05$ & $13.69 \pm 0.05$ & $0.43 \pm 0.07$ & -10.50 & -10.93 & Houck (63) \\
\hline 53013.72 & $0.615 \pm 0.029$ & $0.593 \pm 0.026$ & $14.15 \pm 0.05$ & $13.70 \pm 0.05$ & $0.45 \pm 0.07$ & -10.47 & -10.92 & Gehrz (5) \\
\hline 53208.96 & $0.646 \pm 0.030$ & $0.624 \pm 0.026$ & $14.10 \pm 0.05$ & $13.65 \pm 0.05$ & $0.45 \pm 0.07$ & -10.52 & -10.97 & Gehrz (5) \\
\hline 53233.18 & $0.646 \pm 0.030$ & $0.603 \pm 0.026$ & $14.10 \pm 0.05$ & $13.69 \pm 0.05$ & $0.41 \pm 0.07$ & -10.52 & -10.93 & Gehrz (5) \\
\hline 53391.68 & $0.618 \pm 0.029$ & $0.597 \pm 0.026$ & $14.14 \pm 0.05$ & $13.70 \pm 0.05$ & $0.45 \pm 0.07$ & -10.48 & -10.92 & Gehrz (5) \\
\hline 53607.18 & $0.645 \pm 0.030$ & $0.595 \pm 0.026$ & $14.10 \pm 0.05$ & $13.70 \pm 0.05$ & $0.40 \pm 0.07$ & -10.52 & -10.92 & Gehrz (5) \\
\hline 53770.66 & $0.621 \pm 0.030$ & $0.573 \pm 0.026$ & $14.14 \pm 0.05$ & $13.74 \pm 0.05$ & $0.40 \pm 0.07$ & -10.48 & -10.88 & Gehrz (5) \\
\hline 57111.70 & $0.973 \pm 0.029$ & $0.979 \pm 0.026$ & $13.65 \pm 0.03$ & $13.16 \pm 0.03$ & $0.49 \pm 0.04$ & -10.97 & -11.46 & Kasliwal (11063) \\
\hline 57118.84 & $0.969 \pm 0.029$ & $0.971 \pm 0.025$ & $13.66 \pm 0.03$ & $13.17 \pm 0.03$ & $0.49 \pm 0.04$ & -10.96 & -11.45 & Kasliwal (11063) \\
\hline 57139.32 & $0.952 \pm 0.028$ & $0.942 \pm 0.025$ & $13.68 \pm 0.03$ & $13.20 \pm 0.03$ & $0.47 \pm 0.04$ & -10.94 & -11.42 & Kasliwal (11063) \\
\hline 57314.62 & $0.798 \pm 0.029$ & $0.777 \pm 0.025$ & $13.87 \pm 0.04$ & $13.41 \pm 0.04$ & $0.46 \pm 0.05$ & -10.75 & -11.21 & Kasliwal (11063) \\
\hline 57322.09 & $0.809 \pm 0.029$ & $0.780 \pm 0.025$ & $13.85 \pm 0.04$ & $13.41 \pm 0.04$ & $0.45 \pm 0.05$ & -10.77 & -11.21 & Kasliwal (11063) \\
\hline 57336.48 & $0.780 \pm 0.029$ & $0.770 \pm 0.025$ & $13.89 \pm 0.04$ & $13.42 \pm 0.04$ & $0.47 \pm 0.05$ & -10.73 & -11.20 & Kasliwal (11063) \\
\hline 57491.30 & $0.711 \pm 0.029$ & $0.682 \pm 0.026$ & $13.99 \pm 0.04$ & $13.55 \pm 0.04$ & $0.44 \pm 0.06$ & -10.63 & -11.07 & Kasliwal (11063) \\
\hline \multicolumn{9}{|c|}{ SPIRITS19q } \\
\hline 53285.07 & $0.353 \pm 0.009$ & $0.324 \pm 0.008$ & $14.75 \pm 0.03$ & $14.36 \pm 0.03$ & $0.39 \pm 0.04$ & -12.76 & -13.15 & Van Dyk (226) \\
\hline 53286.42 & $0.346 \pm 0.009$ & $0.328 \pm 0.008$ & $14.77 \pm 0.03$ & $14.35 \pm 0.03$ & $0.42 \pm 0.04$ & -12.74 & -13.16 & Kennicutt (159) \\
\hline 53290.08 & $0.346 \pm 0.009$ & $0.330 \pm 0.009$ & $14.77 \pm 0.03$ & $14.34 \pm 0.03$ & $0.43 \pm 0.04$ & -12.74 & -13.17 & Kennicutt (159) \\
\hline 53310.11 & $0.364 \pm 0.008$ & $0.334 \pm 0.008$ & $14.72 \pm 0.03$ & $14.33 \pm 0.03$ & $0.39 \pm 0.04$ & -12.79 & -13.18 & Van Dyk (226) \\
\hline 53453.94 & $0.340 \pm 0.009$ & $0.311 \pm 0.008$ & $14.79 \pm 0.03$ & $14.40 \pm 0.03$ & $0.39 \pm 0.04$ & -12.72 & -13.11 & Van Dyk (226) \\
\hline 53663.71 & $0.347 \pm 0.008$ & $0.323 \pm 0.008$ & $14.77 \pm 0.03$ & $14.36 \pm 0.03$ & $0.41 \pm 0.04$ & -12.74 & -13.15 & Meikle (20256) \\
\hline 53817.89 & $0.343 \pm 0.008$ & $0.318 \pm 0.008$ & $14.78 \pm 0.03$ & $14.38 \pm 0.03$ & $0.40 \pm 0.04$ & -12.73 & -13.13 & Meikle (20256) \\
\hline 54036.16 & $0.337 \pm 0.009$ & $0.319 \pm 0.008$ & $14.80 \pm 0.03$ & $14.38 \pm 0.03$ & $0.42 \pm 0.04$ & -12.71 & -13.13 & Meikle (30292) \\
\hline 54039.12 & $0.306 \pm 0.012$ & $0.303 \pm 0.014$ & $14.91 \pm 0.04$ & $14.43 \pm 0.05$ & $0.48 \pm 0.07$ & -12.60 & -13.08 & Sugerman (30494) \\
\hline 54192.83 & $0.330 \pm 0.008$ & $0.307 \pm 0.008$ & $14.82 \pm 0.03$ & $14.42 \pm 0.03$ & $0.40 \pm 0.04$ & -12.69 & -13.09 & Sugerman (30494) \\
\hline 54192.84 & $0.328 \pm 0.008$ & $0.310 \pm 0.008$ & $14.83 \pm 0.03$ & $14.41 \pm 0.03$ & $0.42 \pm 0.04$ & -12.68 & -13.10 & Meikle (30292) \\
\hline 54423.33 & $0.336 \pm 0.008$ & $0.325 \pm 0.008$ & $14.80 \pm 0.03$ & $14.36 \pm 0.03$ & $0.44 \pm 0.04$ & -12.71 & -13.15 & Sugerman (30494) \\
\hline 54427.14 & $0.342 \pm 0.008$ & $0.326 \pm 0.008$ & $14.79 \pm 0.03$ & $14.35 \pm 0.03$ & $0.44 \pm 0.04$ & -12.72 & -13.16 & Meixner (40010) \\
\hline 54428.01 & $0.337 \pm 0.009$ & $0.326 \pm 0.008$ & $14.80 \pm 0.03$ & $14.35 \pm 0.03$ & $0.45 \pm 0.04$ & -12.71 & -13.16 & Kotak (40619) \\
\hline 54563.79 & $0.341 \pm 0.008$ & $0.329 \pm 0.008$ & $14.79 \pm 0.03$ & $14.34 \pm 0.03$ & $0.45 \pm 0.04$ & -12.72 & -13.17 & Kotak (40619) \\
\hline 54568.21 & $0.342 \pm 0.008$ & $0.321 \pm 0.008$ & $14.79 \pm 0.03$ & $14.37 \pm 0.03$ & $0.42 \pm 0.04$ & -12.72 & -13.14 & Meixner (40010) \\
\hline 55149.91 & $0.336 \pm 0.009$ & - \pm- & $14.81 \pm 0.03$ & - \pm- & - \pm- & -12.70 & $\ldots$ & Freedman (61002) \\
\hline 55168.68 & $0.345 \pm 0.008$ & - \pm- & $14.78 \pm 0.03$ & - \pm- & - \pm- & -12.73 & $\ldots$ & Freedman (61002) \\
\hline 55185.89 & $0.351 \pm 0.008$ & - \pm- & $14.76 \pm 0.03$ & - \pm- & - \pm- & -12.75 & $\ldots$ & Freedman (61002) \\
\hline 55302.67 & $0.344 \pm 0.008$ & - \pm- & $14.78 \pm 0.03$ & - \pm- & - \pm- & -12.73 & $\ldots$ & Freedman (61002) \\
\hline 55339.50 & $0.350 \pm 0.008$ & - \pm- & $14.76 \pm 0.03$ & - \pm- & - \pm- & -12.75 & $\ldots$ & Freedman (61002) \\
\hline 55890.71 & $0.283 \pm 0.013$ & $0.318 \pm 0.008$ & $14.99 \pm 0.05$ & $14.38 \pm 0.03$ & $0.61 \pm 0.06$ & -12.52 & -13.13 & Kochanek (80015) \\
\hline 56092.54 & $0.356 \pm 0.009$ & $0.342 \pm 0.007$ & $14.74 \pm 0.03$ & $14.30 \pm 0.02$ & $0.44 \pm 0.04$ & -12.77 & -13.21 & Kochanek (80015) \\
\hline 56671.43 & $0.358 \pm 0.009$ & $0.320 \pm 0.008$ & $14.74 \pm 0.03$ & $14.37 \pm 0.03$ & $0.37 \pm 0.04$ & -12.77 & -13.14 & Kasliwal (10136) \\
\hline 56795.32 & $0.341 \pm 0.008$ & $0.319 \pm 0.008$ & $14.79 \pm 0.03$ & $14.38 \pm 0.03$ & $0.41 \pm 0.04$ & -12.72 & -13.13 & Kasliwal (10136) \\
\hline 56827.40 & $0.356 \pm 0.008$ & $0.331 \pm 0.008$ & $14.74 \pm 0.03$ & $14.34 \pm 0.03$ & $0.40 \pm 0.04$ & -12.77 & -13.17 & Kasliwal (10136) \\
\hline 57061.94 & $0.346 \pm 0.008$ & $0.325 \pm 0.008$ & $14.77 \pm 0.03$ & $14.36 \pm 0.03$ & $0.41 \pm 0.04$ & -12.74 & -13.15 & Kasliwal (11063) \\
\hline 57174.60 & $0.353 \pm 0.008$ & $0.332 \pm 0.008$ & $14.75 \pm 0.03$ & $14.33 \pm 0.03$ & $0.42 \pm 0.04$ & -12.76 & -13.18 & Kasliwal (11063) \\
\hline 57180.80 & $0.336 \pm 0.009$ & $0.309 \pm 0.009$ & $14.80 \pm 0.03$ & $14.41 \pm 0.03$ & $0.39 \pm 0.04$ & -12.71 & -13.10 & Kasliwal (11063) \\
\hline 57201.79 & $0.338 \pm 0.008$ & $0.351 \pm 0.008$ & $14.80 \pm 0.03$ & $14.27 \pm 0.03$ & $0.53 \pm 0.04$ & -12.71 & -13.24 & Kasliwal (11063) \\
\hline 57388.85 & $0.370 \pm 0.008$ & $0.334 \pm 0.008$ & $14.70 \pm 0.02$ & $14.33 \pm 0.03$ & $0.37 \pm 0.04$ & -12.81 & -13.18 & Kasliwal (11063) \\
\hline 57395.55 & $0.363 \pm 0.009$ & $0.326 \pm 0.008$ & $14.72 \pm 0.03$ & $14.35 \pm 0.03$ & $0.37 \pm 0.04$ & -12.79 & -13.16 & Kasliwal (11063) \\
\hline 57409.70 & $0.361 \pm 0.008$ & $0.329 \pm 0.008$ & $14.73 \pm 0.03$ & $14.34 \pm 0.03$ & $0.39 \pm 0.04$ & -12.78 & -13.17 & Kasliwal (11063) \\
\hline 57763.83 & $0.364 \pm 0.009$ & $0.336 \pm 0.008$ & $14.72 \pm 0.03$ & $14.32 \pm 0.03$ & $0.40 \pm 0.04$ & -12.79 & -13.19 & Kasliwal (13053) \\
\hline 57929.60 & $0.428 \pm 0.009$ & $0.408 \pm 0.009$ & $14.54 \pm 0.02$ & $14.11 \pm 0.02$ & $0.43 \pm 0.03$ & -12.97 & -13.40 & Kasliwal (13053) \\
\hline 58129.33 & $1.443 \pm 0.010$ & $1.516 \pm 0.009$ & $13.22 \pm 0.01$ & $12.68 \pm 0.01$ & $0.54 \pm 0.01$ & -14.29 & -14.83 & Kasliwal (13053) \\
\hline 58288.90 & $1.738 \pm 0.010$ & $1.980 \pm 0.009$ & $13.02 \pm 0.01$ & $12.39 \pm 0.01$ & $0.63 \pm 0.01$ & -14.49 & -15.12 & Kasliwal (13053) \\
\hline 58507.74 & $1.527 \pm 0.010$ & $1.791 \pm 0.009$ & $13.16 \pm 0.01$ & $12.50 \pm 0.01$ & $0.66 \pm 0.01$ & -14.35 & -15.01 & Kasliwal (14089) \\
\hline 58549.28 & $1.523 \pm 0.010$ & $1.693 \pm 0.008$ & $13.16 \pm 0.01$ & $12.56 \pm 0.01$ & $0.60 \pm 0.01$ & -14.35 & -14.95 & Kasliwal (14089) \\
\hline 58655.40 & $1.277 \pm 0.010$ & $1.528 \pm 0.010$ & $13.36 \pm 0.01$ & $12.68 \pm 0.01$ & $0.68 \pm 0.01$ & -14.15 & -14.83 & Kasliwal (14089) \\
\hline 58697.23 & $1.180 \pm 0.010$ & $1.449 \pm 0.009$ & $13.44 \pm 0.01$ & $12.73 \pm 0.01$ & $0.71 \pm 0.01$ & -14.07 & -14.78 & Kasliwal (14089) \\
\hline \multicolumn{9}{|c|}{ SPIRITS16df } \\
\hline 53285.07 & $0.498 \pm 0.018$ & $0.543 \pm 0.014$ & $14.38 \pm 0.04$ & $13.80 \pm 0.03$ & $0.58 \pm 0.05$ & -13.13 & -13.71 & Van Dyk (226) \\
\hline 53286.42 & $0.471 \pm 0.017$ & $0.515 \pm 0.015$ & $14.44 \pm 0.04$ & $13.86 \pm 0.03$ & $0.58 \pm 0.05$ & -13.07 & -13.65 & Kennicutt (159) \\
\hline 53290.08 & $0.484 \pm 0.017$ & $0.529 \pm 0.015$ & $14.41 \pm 0.04$ & $13.83 \pm 0.03$ & $0.58 \pm 0.05$ & -13.10 & -13.68 & Kennicutt (159) \\
\hline 53310.11 & $0.476 \pm 0.018$ & $0.538 \pm 0.014$ & $14.43 \pm 0.04$ & $13.81 \pm 0.03$ & $0.62 \pm 0.05$ & -13.08 & -13.70 & Van Dyk (226) \\
\hline 53453.94 & $0.461 \pm 0.017$ & $0.502 \pm 0.014$ & $14.46 \pm 0.04$ & $13.89 \pm 0.03$ & $0.57 \pm 0.05$ & -13.05 & -13.62 & Van Dyk (226) \\
\hline
\end{tabular}


Table 2

(Continued)

\begin{tabular}{|c|c|c|c|c|c|c|c|c|}
\hline MJD & $F_{3.6}(\mathrm{mJy})$ & $F_{4.5}(\mathrm{mJy})$ & [3.6] & [4.5] & {$[3.6]-[4.5]$} & $M_{[3.6]}$ & $M_{[4.5]}$ & PI (Prog ID) \\
\hline 53663.71 & $0.475 \pm 0.017$ & $0.556 \pm 0.014$ & $14.43 \pm 0.04$ & $13.77 \pm 0.03$ & $0.66 \pm 0.05$ & -13.08 & -13.74 & Meikle (20256) \\
\hline 53817.89 & $0.466 \pm 0.017$ & $0.531 \pm 0.014$ & $14.45 \pm 0.04$ & $13.82 \pm 0.03$ & $0.63 \pm 0.05$ & -13.06 & -13.69 & Meikle (20256) \\
\hline 54036.16 & $0.465 \pm 0.017$ & $0.540 \pm 0.015$ & $14.45 \pm 0.04$ & $13.81 \pm 0.03$ & $0.64 \pm 0.05$ & -13.06 & -13.70 & Meikle (30292) \\
\hline 54039.12 & $0.429 \pm 0.019$ & $0.512 \pm 0.020$ & $14.54 \pm 0.05$ & $13.86 \pm 0.04$ & $0.68 \pm 0.06$ & -12.97 & -13.65 & Sugerman (30494) \\
\hline 54192.83 & $0.491 \pm 0.018$ & $0.536 \pm 0.014$ & $14.39 \pm 0.04$ & $13.81 \pm 0.03$ & $0.58 \pm 0.05$ & -13.12 & -13.70 & Sugerman (30494) \\
\hline 54192.84 & $0.484 \pm 0.017$ & $0.530 \pm 0.014$ & $14.41 \pm 0.04$ & $13.83 \pm 0.03$ & $0.58 \pm 0.05$ & -13.10 & -13.68 & Meikle (30292) \\
\hline 54423.33 & $0.469 \pm 0.017$ & $0.533 \pm 0.014$ & $14.44 \pm 0.04$ & $13.82 \pm 0.03$ & $0.62 \pm 0.05$ & -13.07 & -13.69 & Sugerman (30494) \\
\hline 54427.14 & $0.476 \pm 0.017$ & $0.538 \pm 0.015$ & $14.43 \pm 0.04$ & $13.81 \pm 0.03$ & $0.62 \pm 0.05$ & -13.08 & -13.70 & Meixner (40010) \\
\hline 54428.01 & $0.462 \pm 0.017$ & $0.525 \pm 0.014$ & $14.46 \pm 0.04$ & $13.83 \pm 0.03$ & $0.63 \pm 0.05$ & -13.05 & -13.68 & Kotak (40619) \\
\hline 54563.79 & $0.476 \pm 0.018$ & $0.511 \pm 0.015$ & $14.43 \pm 0.04$ & $13.87 \pm 0.03$ & $0.56 \pm 0.05$ & -13.08 & -13.64 & Kotak (40619) \\
\hline 54568.21 & $0.479 \pm 0.017$ & $0.545 \pm 0.014$ & $14.42 \pm 0.04$ & $13.79 \pm 0.03$ & $0.63 \pm 0.05$ & -13.09 & -13.72 & Meixner(40010) \\
\hline 55890.71 & $0.470 \pm 0.018$ & $0.533 \pm 0.013$ & $14.44 \pm 0.04$ & $13.82 \pm 0.03$ & $0.62 \pm 0.05$ & -13.07 & -13.69 & Kochanek (80015) \\
\hline 56092.54 & $0.538 \pm 0.017$ & $0.572 \pm 0.014$ & $14.29 \pm 0.03$ & $13.74 \pm 0.03$ & $0.55 \pm 0.04$ & -13.22 & -13.77 & Kochanek (80015) \\
\hline 56650.66 & $0.471 \pm 0.018$ & $0.524 \pm 0.014$ & $14.44 \pm 0.04$ & $13.84 \pm 0.03$ & $0.60 \pm 0.05$ & -13.07 & -13.67 & Kochanek (10001) \\
\hline 56671.43 & $0.492 \pm 0.017$ & $0.537 \pm 0.015$ & $14.39 \pm 0.04$ & $13.81 \pm 0.03$ & $0.58 \pm 0.05$ & -13.12 & -13.70 & Kasliwal (10136) \\
\hline 56795.32 & $0.466 \pm 0.017$ & $0.511 \pm 0.014$ & $14.45 \pm 0.04$ & $13.87 \pm 0.03$ & $0.58 \pm 0.05$ & -13.06 & -13.64 & Kasliwal (10136) \\
\hline 56827.40 & $0.515 \pm 0.017$ & $0.539 \pm 0.014$ & $14.34 \pm 0.04$ & $13.81 \pm 0.03$ & $0.53 \pm 0.05$ & -13.17 & -13.70 & Kasliwal (10136) \\
\hline 57061.94 & $0.531 \pm 0.018$ & $0.579 \pm 0.014$ & $14.31 \pm 0.04$ & $13.73 \pm 0.03$ & $0.58 \pm 0.05$ & -13.20 & -13.78 & Kasliwal (11063) \\
\hline 57174.60 & $0.490 \pm 0.017$ & $0.528 \pm 0.015$ & $14.40 \pm 0.04$ & $13.83 \pm 0.03$ & $0.57 \pm 0.05$ & -13.11 & -13.68 & Kasliwal (11063) \\
\hline 57180.80 & $0.519 \pm 0.017$ & $0.562 \pm 0.014$ & $14.33 \pm 0.04$ & $13.76 \pm 0.03$ & $0.57 \pm 0.04$ & -13.18 & -13.75 & Kasliwal (11063) \\
\hline 57201.79 & $0.525 \pm 0.018$ & $0.574 \pm 0.015$ & $14.32 \pm 0.04$ & $13.74 \pm 0.03$ & $0.58 \pm 0.05$ & -13.19 & -13.77 & Kasliwal (11063) \\
\hline 57388.85 & $0.512 \pm 0.017$ & $0.562 \pm 0.014$ & $14.35 \pm 0.04$ & $13.76 \pm 0.03$ & $0.59 \pm 0.05$ & -13.16 & -13.75 & Kasliwal (11063) \\
\hline 57395.55 & $0.494 \pm 0.017$ & $0.543 \pm 0.014$ & $14.39 \pm 0.04$ & $13.80 \pm 0.03$ & $0.59 \pm 0.05$ & -13.12 & -13.71 & Kasliwal (11063) \\
\hline 57409.70 & $0.499 \pm 0.017$ & $0.573 \pm 0.015$ & $14.38 \pm 0.04$ & $13.74 \pm 0.03$ & $0.64 \pm 0.05$ & -13.13 & -13.77 & Kasliwal (11063) \\
\hline 57763.83 & $0.446 \pm 0.017$ & $0.510 \pm 0.015$ & $14.50 \pm 0.04$ & $13.87 \pm 0.03$ & $0.63 \pm 0.05$ & -13.01 & -13.64 & Kasliwal (13053) \\
\hline 57929.60 & $0.482 \pm 0.017$ & $0.541 \pm 0.015$ & $14.41 \pm 0.04$ & $13.80 \pm 0.03$ & $0.61 \pm 0.05$ & -13.10 & -13.71 & Kasliwal (13053) \\
\hline 58129.33 & $0.480 \pm 0.017$ & $0.536 \pm 0.015$ & $14.42 \pm 0.04$ & $13.81 \pm 0.03$ & $0.61 \pm 0.05$ & -13.09 & -13.70 & Kasliwal (13053) \\
\hline 58288.90 & $0.446 \pm 0.017$ & $0.486 \pm 0.015$ & $14.50 \pm 0.04$ & $13.92 \pm 0.03$ & $0.58 \pm 0.05$ & -13.01 & -13.59 & Kasliwal (13053) \\
\hline 58507.74 & $0.494 \pm 0.017$ & $0.543 \pm 0.014$ & $14.39 \pm 0.04$ & $13.80 \pm 0.03$ & $0.59 \pm 0.05$ & -13.12 & -13.71 & Kasliwal (14089) \\
\hline 58549.28 & $0.494 \pm 0.018$ & $0.549 \pm 0.014$ & $14.39 \pm 0.04$ & $13.79 \pm 0.03$ & $0.60 \pm 0.05$ & -13.12 & -13.72 & Kasliwal (14089) \\
\hline 58655.40 & $0.466 \pm 0.017$ & $0.492 \pm 0.015$ & $14.45 \pm 0.04$ & $13.91 \pm 0.03$ & $0.54 \pm 0.05$ & -13.06 & -13.60 & Kasliwal (14089) \\
\hline 58697.23 & $0.455 \pm 0.018$ & $0.513 \pm 0.015$ & $14.48 \pm 0.04$ & $13.86 \pm 0.03$ & $0.62 \pm 0.05$ & -13.03 & -13.65 & Kasliwal (14089) \\
\hline \multicolumn{9}{|c|}{ SPIRITS14apu } \\
\hline 53072.09 & $0.395 \pm 0.010$ & $0.430 \pm 0.009$ & $14.63 \pm 0.03$ & $14.05 \pm 0.02$ & $0.58 \pm 0.04$ & -14.41 & -14.99 & Rieke (60) \\
\hline 53072.49 & $0.408 \pm 0.009$ & $0.458 \pm 0.008$ & $14.59 \pm 0.02$ & $13.98 \pm 0.02$ & $0.61 \pm 0.03$ & -14.45 & -15.06 & Rieke (60) \\
\hline 55960.72 & $0.549 \pm 0.009$ & - \pm- & $14.27 \pm 0.02$ & - \pm- & - \pm- & -14.77 & $\ldots$ & Kasliwal (80196) \\
\hline 55980.99 & $0.556 \pm 0.009$ & - \pm- & $14.26 \pm 0.02$ & - \pm- & - \pm- & -14.78 & $\cdots$ & Kasliwal (80196) \\
\hline 56165.01 & - \pm- & $0.612 \pm 0.008$ & - \pm- & $13.67 \pm 0.01$ & - \pm- & $\ldots$ & -15.37 & Garnavich (80126) \\
\hline 56337.07 & $0.563 \pm 0.010$ & - \pm- & $14.25 \pm 0.02$ & - \pm- & - \pm- & -14.79 & $\ldots$ & Kasliwal (90240) \\
\hline 56348.11 & $0.565 \pm 0.010$ & - \pm- & $14.24 \pm 0.02$ & - \pm- & - \pm- & -14.80 & $\cdots$ & Garnavich (80126) \\
\hline 56393.76 & $0.614 \pm 0.010$ & - \pm- & $14.15 \pm 0.02$ & - \pm- & - \pm- & -14.89 & $\cdots$ & Kasliwal (90240) \\
\hline 56516.35 & - \pm- & $0.645 \pm 0.008$ & - \pm- & $13.61 \pm 0.01$ & - \pm- & $\ldots$ & -15.43 & Kasliwal (90240) \\
\hline 56742.84 & $0.508 \pm 0.009$ & $0.617 \pm 0.007$ & $14.36 \pm 0.02$ & $13.66 \pm 0.01$ & $0.70 \pm 0.02$ & -14.68 & -15.38 & Kasliwal (10136) \\
\hline 56771.83 & $0.512 \pm 0.009$ & $0.622 \pm 0.007$ & $14.35 \pm 0.02$ & $13.65 \pm 0.01$ & $0.70 \pm 0.02$ & -14.69 & -15.39 & Kasliwal (10136) \\
\hline 56902.01 & $0.443 \pm 0.010$ & $0.572 \pm 0.008$ & $14.50 \pm 0.02$ & $13.74 \pm 0.02$ & $0.76 \pm 0.03$ & -14.54 & -15.30 & Kasliwal (10136) \\
\hline 57136.69 & $0.442 \pm 0.009$ & $0.546 \pm 0.008$ & $14.51 \pm 0.02$ & $13.79 \pm 0.02$ & $0.72 \pm 0.03$ & -14.53 & -15.25 & Kasliwal (11063) \\
\hline 57144.06 & $0.425 \pm 0.009$ & $0.522 \pm 0.008$ & $14.55 \pm 0.02$ & $13.84 \pm 0.02$ & $0.71 \pm 0.03$ & -14.49 & -15.20 & Kasliwal (11063) \\
\hline 57150.17 & $0.454 \pm 0.010$ & $0.549 \pm 0.008$ & $14.48 \pm 0.02$ & $13.79 \pm 0.02$ & $0.69 \pm 0.03$ & -14.56 & -15.25 & Kasliwal (11063) \\
\hline 57163.71 & $0.457 \pm 0.009$ & $0.558 \pm 0.008$ & $14.47 \pm 0.02$ & $13.77 \pm 0.02$ & $0.70 \pm 0.03$ & -14.57 & -15.27 & Kasliwal (11063) \\
\hline 57191.82 & $0.465 \pm 0.009$ & $0.574 \pm 0.008$ & $14.45 \pm 0.02$ & $13.74 \pm 0.01$ & $0.71 \pm 0.03$ & -14.59 & -15.30 & Kasliwal (11063) \\
\hline 57220.79 & $0.461 \pm 0.009$ & $0.585 \pm 0.007$ & $14.46 \pm 0.02$ & $13.72 \pm 0.01$ & $0.74 \pm 0.02$ & -14.58 & -15.32 & Kasliwal (11063) \\
\hline 57247.82 & $0.439 \pm 0.009$ & $0.561 \pm 0.007$ & $14.51 \pm 0.02$ & $13.76 \pm 0.01$ & $0.75 \pm 0.03$ & -14.53 & -15.28 & Kasliwal (11063) \\
\hline 57486.85 & $0.487 \pm 0.009$ & $0.632 \pm 0.007$ & $14.40 \pm 0.02$ & $13.63 \pm 0.01$ & $0.77 \pm 0.02$ & -14.64 & -15.41 & Kasliwal (11063) \\
\hline 57843.93 & $0.552 \pm 0.010$ & $0.648 \pm 0.008$ & $14.27 \pm 0.02$ & $13.61 \pm 0.01$ & $0.66 \pm 0.02$ & -14.77 & -15.43 & Kasliwal (13053) \\
\hline 57926.90 & $0.585 \pm 0.010$ & $0.713 \pm 0.009$ & $14.20 \pm 0.02$ & $13.50 \pm 0.01$ & $0.70 \pm 0.02$ & -14.84 & -15.54 & Kasliwal (13053) \\
\hline 58009.67 & $0.509 \pm 0.009$ & $0.609 \pm 0.008$ & $14.35 \pm 0.02$ & $13.68 \pm 0.01$ & $0.67 \pm 0.02$ & -14.69 & -15.36 & Kasliwal (13053) \\
\hline 58232.95 & $0.557 \pm 0.009$ & $0.657 \pm 0.007$ & $14.26 \pm 0.02$ & $13.59 \pm 0.01$ & $0.67 \pm 0.02$ & -14.78 & -15.45 & Kasliwal (13053) \\
\hline 58292.87 & $0.550 \pm 0.010$ & $0.666 \pm 0.008$ & $14.27 \pm 0.02$ & $13.58 \pm 0.01$ & $0.69 \pm 0.02$ & -14.77 & -15.46 & Kasliwal (13053) \\
\hline 58380.22 & $0.573 \pm 0.009$ & $0.685 \pm 0.008$ & $14.23 \pm 0.02$ & $13.55 \pm 0.01$ & $0.68 \pm 0.02$ & -14.81 & -15.49 & Kasliwal (13053) \\
\hline 58572.08 & $0.529 \pm 0.009$ & $0.644 \pm 0.008$ & $14.31 \pm 0.02$ & $13.61 \pm 0.01$ & $0.70 \pm 0.02$ & -14.73 & -15.43 & Kasliwal (14089) \\
\hline 58614.39 & $0.523 \pm 0.009$ & $0.633 \pm 0.008$ & $14.33 \pm 0.02$ & $13.63 \pm 0.01$ & $0.70 \pm 0.02$ & -14.71 & -15.41 & Kasliwal (14089) \\
\hline 58655.68 & $0.587 \pm 0.009$ & $0.682 \pm 0.008$ & $14.20 \pm 0.02$ & $13.55 \pm 0.01$ & $0.65 \pm 0.02$ & -14.84 & -15.49 & Kasliwal (14089) \\
\hline 58697.50 & $0.585 \pm 0.010$ & $0.701 \pm 0.008$ & $14.20 \pm 0.02$ & $13.52 \pm 0.01$ & $0.68 \pm 0.02$ & -14.84 & -15.52 & Kasliwal (14089) \\
\hline 58740.01 & $0.533 \pm 0.009$ & $0.648 \pm 0.008$ & $14.30 \pm 0.02$ & $13.61 \pm 0.01$ & $0.69 \pm 0.02$ & -14.74 & -15.43 & Kasliwal (14089) \\
\hline
\end{tabular}


Table 2

(Continued)

\begin{tabular}{|c|c|c|c|c|c|c|c|c|}
\hline MJD & $F_{3.6}(\mathrm{mJy})$ & $F_{4.5}(\mathrm{mJy})$ & {$[3.6]$} & {$[4.5]$} & {$[3.6]-[4.5]$} & $M_{[3.6]}$ & $M_{[4.5]}$ & PI (Prog ID) \\
\hline 58781.31 & $0.624 \pm 0.010$ & $0.711 \pm 0.008$ & $14.13 \pm 0.02$ & $13.51 \pm 0.01$ & $0.62 \pm 0.02$ & -14.91 & -15.53 & Kasliwal (14089) \\
\hline \multicolumn{9}{|c|}{ SPIRITS18hb } \\
\hline 53166.76 & $0.428 \pm 0.027$ & $0.355 \pm 0.022$ & $14.54 \pm 0.07$ & $14.26 \pm 0.07$ & $0.28 \pm 0.10$ & -14.90 & -15.18 & Kennicutt (159) \\
\hline 53260.29 & - \pm- & $0.335 \pm 0.022$ & - \pm- & $14.32 \pm 0.07$ & - \pm- & $\ldots$ & -15.12 & Meikle (3248) \\
\hline 53334.74 & $0.388 \pm 0.026$ & $0.328 \pm 0.022$ & $14.65 \pm 0.07$ & $14.35 \pm 0.07$ & $0.30 \pm 0.10$ & -14.79 & -15.09 & Kennicutt (159) \\
\hline 53571.24 & - \pm- & $0.318 \pm 0.021$ & - \pm- & $14.38 \pm 0.07$ & - \pm- & $\ldots$ & -15.06 & Meikle (20256) \\
\hline 53630.81 & - \pm- & $0.337 \pm 0.021$ & - \pm- & $14.32 \pm 0.07$ & - \pm- & $\ldots$ & -15.12 & Sugerman (20320) \\
\hline 53676.05 & - \pm- & $0.364 \pm 0.022$ & - \pm- & $14.23 \pm 0.06$ & - \pm- & $\ldots$ & -15.21 & Meikle (20256) \\
\hline 53734.89 & - \pm- & $0.334 \pm 0.025$ & - \pm- & $14.33 \pm 0.08$ & - \pm- & $\ldots$ & -15.11 & Sugerman (20320) \\
\hline 54065.89 & - \pm- & $0.361 \pm 0.022$ & - \pm- & $14.24 \pm 0.07$ & - \pm- & $\ldots$ & -15.20 & Meikle (30292) \\
\hline 54098.03 & - \pm- & $0.367 \pm 0.021$ & - \pm- & $14.23 \pm 0.06$ & - \pm- & $\ldots$ & -15.21 & Sugerman (30494) \\
\hline 54284.97 & $0.419 \pm 0.026$ & - \pm- & $14.56 \pm 0.07$ & - \pm- & - \pm- & -14.88 & $\ldots$ & Sugerman (30494) \\
\hline 54461.07 & - \pm- & $0.400 \pm 0.021$ & - \pm- & $14.13 \pm 0.06$ & - \pm- & $\ldots$ & -15.31 & Meixner (40010) \\
\hline 54492.85 & - \pm- & $0.380 \pm 0.022$ & - \pm- & $14.19 \pm 0.06$ & - \pm- & $\ldots$ & -15.25 & Kotak (40619) \\
\hline 54665.79 & $0.459 \pm 0.025$ & - \pm- & $14.47 \pm 0.06$ & - \pm- & - \pm- & -14.97 & $\ldots$ & Meixner (40010) \\
\hline 55049.01 & $0.400 \pm 0.024$ & - \pm- & $14.62 \pm 0.07$ & - \pm- & - \pm- & -14.82 & $\ldots$ & Andrews (60071) \\
\hline 55201.62 & - \pm- & $0.373 \pm 0.021$ & - \pm- & $14.21 \pm 0.06$ & - \pm- & $\ldots$ & -15.23 & Andrews (60071) \\
\hline 55421.58 & $0.438 \pm 0.025$ & - \pm- & $14.52 \pm 0.06$ & - \pm- & - \pm- & -14.92 & $\ldots$ & Andrews (70008) \\
\hline 55531.43 & - \pm- & $0.374 \pm 0.020$ & - \pm- & $14.21 \pm 0.06$ & - \pm- & $\ldots$ & -15.23 & Kochanek (70040) \\
\hline 55566.90 & - \pm- & $0.376 \pm 0.021$ & - \pm- & $14.20 \pm 0.06$ & - \pm- & $\ldots$ & -15.24 & Andrews (70008) \\
\hline 55769.87 & $0.455 \pm 0.026$ & - \pm- & $14.48 \pm 0.06$ & - \pm- & - \pm- & -14.96 & $\ldots$ & Kochanek (80015) \\
\hline 55774.83 & $0.461 \pm 0.026$ & - \pm- & $14.46 \pm 0.06$ & - \pm- & - \pm- & -14.98 & $\cdots$ & Andrews (80131) \\
\hline 55939.12 & - \pm- & $0.379 \pm 0.021$ & - \pm- & $14.19 \pm 0.06$ & - \pm- & $\ldots$ & -15.25 & Andrews (80131) \\
\hline 56521.15 & $0.459 \pm 0.026$ & - \pm- & $14.47 \pm 0.06$ & - \pm- & - \pm- & -14.97 & $\ldots$ & Andrews (90178) \\
\hline 56660.59 & - \pm- & $0.392 \pm 0.021$ & - \pm- & $14.15 \pm 0.06$ & - \pm- & $\ldots$ & -15.29 & Kochanek (10081) \\
\hline 56705.62 & - \pm- & $0.383 \pm 0.021$ & - \pm- & $14.18 \pm 0.06$ & - \pm- & $\ldots$ & -15.26 & Sugerman (10002) \\
\hline 56742.56 & $0.461 \pm 0.026$ & $0.403 \pm 0.021$ & $14.46 \pm 0.06$ & $14.12 \pm 0.06$ & $0.34 \pm 0.08$ & -14.98 & -15.32 & Kasliwal (10136) \\
\hline 56916.20 & $0.468 \pm 0.026$ & $0.394 \pm 0.021$ & $14.45 \pm 0.06$ & $14.15 \pm 0.06$ & $0.30 \pm 0.08$ & -14.99 & -15.29 & Kasliwal (10136) \\
\hline 56945.57 & $0.481 \pm 0.026$ & $0.396 \pm 0.021$ & $14.42 \pm 0.06$ & $14.14 \pm 0.06$ & $0.28 \pm 0.08$ & -15.02 & -15.30 & Kasliwal (10136) \\
\hline 57293.99 & $0.446 \pm 0.023$ & $0.376 \pm 0.020$ & $14.50 \pm 0.06$ & $14.20 \pm 0.06$ & $0.30 \pm 0.08$ & -14.94 & -15.24 & Kasliwal (11063) \\
\hline 57322.05 & $0.495 \pm 0.024$ & $0.435 \pm 0.022$ & $14.38 \pm 0.05$ & $14.04 \pm 0.05$ & $0.34 \pm 0.08$ & -15.06 & -15.40 & Kasliwal (11063) \\
\hline 57351.45 & $0.438 \pm 0.024$ & $0.447 \pm 0.021$ & $14.52 \pm 0.06$ & $14.01 \pm 0.05$ & $0.51 \pm 0.08$ & -14.92 & -15.43 & Kasliwal (11063) \\
\hline 57673.30 & $0.490 \pm 0.027$ & $0.432 \pm 0.022$ & $14.39 \pm 0.06$ & $14.05 \pm 0.06$ & $0.34 \pm 0.08$ & -15.05 & -15.39 & Kasliwal (13053) \\
\hline 58080.79 & $0.499 \pm 0.026$ & $0.467 \pm 0.022$ & $14.38 \pm 0.06$ & $13.96 \pm 0.05$ & $0.42 \pm 0.08$ & -15.06 & -15.48 & Krafton (13239) \\
\hline 58178.15 & $0.508 \pm 0.026$ & $0.470 \pm 0.021$ & $14.36 \pm 0.06$ & $13.96 \pm 0.05$ & $0.40 \pm 0.07$ & -15.08 & -15.48 & Krafton (13239) \\
\hline 58381.85 & $0.524 \pm 0.026$ & $0.493 \pm 0.022$ & $14.32 \pm 0.05$ & $13.90 \pm 0.05$ & $0.42 \pm 0.07$ & -15.12 & -15.54 & Krafton (13239) \\
\hline 58404.83 & $0.528 \pm 0.024$ & $0.471 \pm 0.022$ & $14.31 \pm 0.05$ & $13.95 \pm 0.05$ & $0.36 \pm 0.07$ & -15.13 & -15.49 & Fox (14098) \\
\hline 58449.30 & $0.507 \pm 0.025$ & $0.495 \pm 0.022$ & $14.36 \pm 0.05$ & $13.90 \pm 0.05$ & $0.46 \pm 0.07$ & -15.08 & -15.54 & Kasliwal (14089) \\
\hline 58515.57 & $0.533 \pm 0.026$ & $0.508 \pm 0.023$ & $14.31 \pm 0.05$ & $13.87 \pm 0.05$ & $0.44 \pm 0.07$ & -15.13 & -15.57 & Krafton (13239) \\
\hline 58529.84 & $0.542 \pm 0.027$ & $0.504 \pm 0.022$ & $14.29 \pm 0.05$ & $13.88 \pm 0.05$ & $0.41 \pm 0.07$ & -15.15 & -15.56 & Kasliwal (14089) \\
\hline 58607.95 & $0.537 \pm 0.025$ & $0.536 \pm 0.021$ & $14.30 \pm 0.05$ & $13.81 \pm 0.04$ & $0.49 \pm 0.07$ & -15.14 & -15.63 & Kasliwal (14089) \\
\hline 58795.40 & $0.463 \pm 0.026$ & $0.444 \pm 0.022$ & $14.46 \pm 0.06$ & $14.02 \pm 0.05$ & $0.44 \pm 0.08$ & -14.98 & -15.42 & Kasliwal (14089) \\
\hline \multicolumn{9}{|c|}{ SPIRITS14bqe } \\
\hline 52994.85 & - \pm- & $0.115 \pm 0.001$ & - \pm- & $15.48 \pm 0.01$ & - \pm- & $\ldots$ & -8.82 & Gehrz (128) \\
\hline 53727.66 & $0.070 \pm 0.001$ & - \pm- & $16.51 \pm 0.02$ & - \pm- & - \pm- & -7.79 & $\ldots$ & Gehrz (128) \\
\hline 55222.14 & $0.087 \pm 0.001$ & $0.149 \pm 0.001$ & $16.28 \pm 0.01$ & $15.21 \pm 0.01$ & $1.07 \pm 0.01$ & -8.02 & -9.09 & Freedman (61001) \\
\hline 55233.99 & $0.100 \pm 0.001$ & $0.182 \pm 0.001$ & $16.12 \pm 0.01$ & $14.98 \pm 0.01$ & $1.14 \pm 0.01$ & -8.18 & -9.32 & Freedman (61001) \\
\hline 55241.02 & $0.107 \pm 0.001$ & $0.189 \pm 0.001$ & $16.05 \pm 0.01$ & $14.95 \pm 0.01$ & $1.10 \pm 0.01$ & -8.25 & -9.35 & Freedman (61001) \\
\hline 55252.40 & $0.100 \pm 0.001$ & $0.172 \pm 0.001$ & $16.12 \pm 0.01$ & $15.05 \pm 0.01$ & $1.07 \pm 0.01$ & -8.18 & -9.25 & Freedman (61001) \\
\hline 55428.67 & $0.090 \pm 0.001$ & $0.147 \pm 0.001$ & $16.23 \pm 0.01$ & $15.22 \pm 0.01$ & $1.01 \pm 0.01$ & -8.07 & -9.08 & Freedman (61001) \\
\hline 55436.71 & $0.089 \pm 0.001$ & $0.151 \pm 0.001$ & $16.25 \pm 0.02$ & $15.19 \pm 0.01$ & $1.06 \pm 0.01$ & -8.05 & -9.11 & Freedman (61001) \\
\hline 55446.80 & $0.092 \pm 0.001$ & $0.143 \pm 0.001$ & $16.22 \pm 0.02$ & $15.25 \pm 0.01$ & $0.97 \pm 0.01$ & -8.08 & -9.05 & Freedman (61001) \\
\hline 55456.01 & $0.091 \pm 0.001$ & $0.146 \pm 0.001$ & $16.23 \pm 0.02$ & $15.22 \pm 0.01$ & $1.01 \pm 0.01$ & -8.07 & -9.08 & Freedman (61001) \\
\hline 55595.93 & $0.079 \pm 0.001$ & $0.120 \pm 0.001$ & $16.38 \pm 0.02$ & $15.44 \pm 0.01$ & $0.94 \pm 0.02$ & -7.92 & -8.86 & Freedman (61001) \\
\hline 55605.12 & $0.079 \pm 0.001$ & $0.113 \pm 0.001$ & $16.38 \pm 0.02$ & $15.50 \pm 0.01$ & $0.88 \pm 0.02$ & -7.92 & -8.80 & Freedman (61001) \\
\hline 55615.06 & $0.076 \pm 0.001$ & $0.117 \pm 0.001$ & $16.41 \pm 0.02$ & $15.46 \pm 0.01$ & $0.95 \pm 0.02$ & -7.89 & -8.84 & Freedman (61001) \\
\hline 55625.55 & $0.075 \pm 0.001$ & $0.115 \pm 0.001$ & $16.43 \pm 0.02$ & $15.48 \pm 0.01$ & $0.95 \pm 0.02$ & -7.87 & -8.82 & Freedman (61001) \\
\hline 55825.25 & $0.070 \pm 0.001$ & $0.104 \pm 0.001$ & $16.52 \pm 0.02$ & $15.60 \pm 0.01$ & $0.92 \pm 0.02$ & -7.78 & -8.70 & Boyer (80063) \\
\hline 55977.41 & $0.065 \pm 0.001$ & $0.095 \pm 0.001$ & $16.59 \pm 0.02$ & $15.69 \pm 0.01$ & $0.90 \pm 0.02$ & -7.71 & -8.61 & Boyer (80063) \\
\hline 56714.37 & $0.064 \pm 0.001$ & - \pm- & $16.60 \pm 0.02$ & - \pm- & - \pm- & -7.70 & $\ldots$ & Colbert (90230) \\
\hline 56748.93 & $0.062 \pm 0.001$ & $0.089 \pm 0.001$ & $16.65 \pm 0.02$ & $15.77 \pm 0.01$ & $0.88 \pm 0.02$ & -7.65 & -8.53 & Kasliwal (10136) \\
\hline 56922.78 & $0.065 \pm 0.001$ & $0.090 \pm 0.001$ & $16.59 \pm 0.02$ & $15.76 \pm 0.01$ & $0.83 \pm 0.02$ & -7.71 & -8.54 & Kasliwal (10136) \\
\hline 56951.33 & $0.063 \pm 0.001$ & $0.081 \pm 0.001$ & $16.62 \pm 0.02$ & $15.86 \pm 0.01$ & $0.76 \pm 0.02$ & -7.68 & -8.44 & Kasliwal (10136) \\
\hline
\end{tabular}


Table 2

(Continued)

\begin{tabular}{|c|c|c|c|c|c|c|c|c|}
\hline MJD & $F_{3.6}(\mathrm{mJy})$ & $F_{4.5}(\mathrm{mJy})$ & [3.6] & [4.5] & {$[3.6]-[4.5]$} & $M_{[3.6]}$ & $M_{[4.5]}$ & PI (Prog ID) \\
\hline 57121.20 & $0.062 \pm 0.001$ & $0.082 \pm 0.001$ & $16.63 \pm 0.02$ & $15.85 \pm 0.02$ & $0.78 \pm 0.02$ & -7.67 & -8.45 & Kasliwal (11063) \\
\hline 57300.46 & $0.064 \pm 0.001$ & $0.084 \pm 0.001$ & $16.61 \pm 0.02$ & $15.82 \pm 0.01$ & $0.79 \pm 0.02$ & -7.69 & -8.48 & Kasliwal (11063) \\
\hline 57305.62 & $0.063 \pm 0.001$ & $0.094 \pm 0.001$ & $16.62 \pm 0.02$ & $15.71 \pm 0.01$ & $0.91 \pm 0.02$ & -7.68 & -8.59 & Kasliwal (11063) \\
\hline 57320.45 & $0.063 \pm 0.001$ & $0.083 \pm 0.001$ & $16.63 \pm 0.02$ & $15.84 \pm 0.01$ & $0.79 \pm 0.02$ & -7.67 & -8.46 & Kasliwal (11063) \\
\hline 57462.10 & $0.064 \pm 0.001$ & $0.083 \pm 0.001$ & $16.60 \pm 0.02$ & $15.83 \pm 0.01$ & $0.77 \pm 0.02$ & -7.70 & -8.47 & Kasliwal (11063) \\
\hline 57469.00 & $0.062 \pm 0.001$ & $0.081 \pm 0.001$ & $16.63 \pm 0.02$ & $15.87 \pm 0.01$ & $0.76 \pm 0.02$ & -7.67 & -8.43 & Kasliwal (11063) \\
\hline 57488.83 & $0.064 \pm 0.001$ & $0.082 \pm 0.001$ & $16.61 \pm 0.02$ & $15.86 \pm 0.01$ & $0.75 \pm 0.02$ & -7.69 & -8.44 & Kasliwal (11063) \\
\hline 57673.33 & $0.062 \pm 0.001$ & $0.081 \pm 0.001$ & $16.64 \pm 0.02$ & $15.86 \pm 0.01$ & $0.78 \pm 0.02$ & -7.66 & -8.44 & Kasliwal (13053) \\
\hline 57852.92 & $0.061 \pm 0.001$ & $0.079 \pm 0.001$ & $16.67 \pm 0.02$ & $15.90 \pm 0.01$ & $0.77 \pm 0.02$ & -7.63 & -8.40 & Kasliwal (13053) \\
\hline 58045.60 & $0.062 \pm 0.001$ & $0.081 \pm 0.001$ & $16.63 \pm 0.02$ & $15.86 \pm 0.01$ & $0.77 \pm 0.02$ & -7.67 & -8.44 & Kasliwal (13053) \\
\hline 58242.81 & $0.091 \pm 0.001$ & $0.150 \pm 0.001$ & $16.22 \pm 0.02$ & $15.20 \pm 0.01$ & $1.02 \pm 0.01$ & -8.08 & -9.10 & Kasliwal (13053) \\
\hline 58443.65 & $0.104 \pm 0.001$ & $0.188 \pm 0.001$ & $16.08 \pm 0.01$ & $14.95 \pm 0.01$ & $1.13 \pm 0.01$ & -8.22 & -9.35 & Kasliwal (14089) \\
\hline 58581.44 & $0.074 \pm 0.001$ & $0.111 \pm 0.001$ & $16.45 \pm 0.02$ & $15.52 \pm 0.01$ & $0.93 \pm 0.02$ & -7.85 & -8.78 & Kasliwal (14089) \\
\hline 58607.97 & $0.074 \pm 0.001$ & $0.109 \pm 0.001$ & $16.44 \pm 0.02$ & $15.54 \pm 0.01$ & $0.90 \pm 0.02$ & -7.86 & -8.76 & Kasliwal (14089) \\
\hline 58795.85 & $0.067 \pm 0.001$ & $0.098 \pm 0.001$ & $16.56 \pm 0.02$ & $15.66 \pm 0.01$ & $0.90 \pm 0.02$ & -7.74 & -8.64 & Kasliwal (14089) \\
\hline
\end{tabular}

Note. Spitzer/IRAC 3.6 and $4.5 \mu \mathrm{m}$ photometry (in Jy and magnitudes), [3.6] - [4.5] color, and absolute magnitudes with $1 \sigma$ uncertainties of the six dust-forming WC candidates SPIRITS 16ln, 19q, 16df, 14apu, 18hb, and 14bqe. The PI names and program ID numbers of the Spitzer/IRAC programs associated with the observations are also provided. All magnitudes are given in the Vega system.

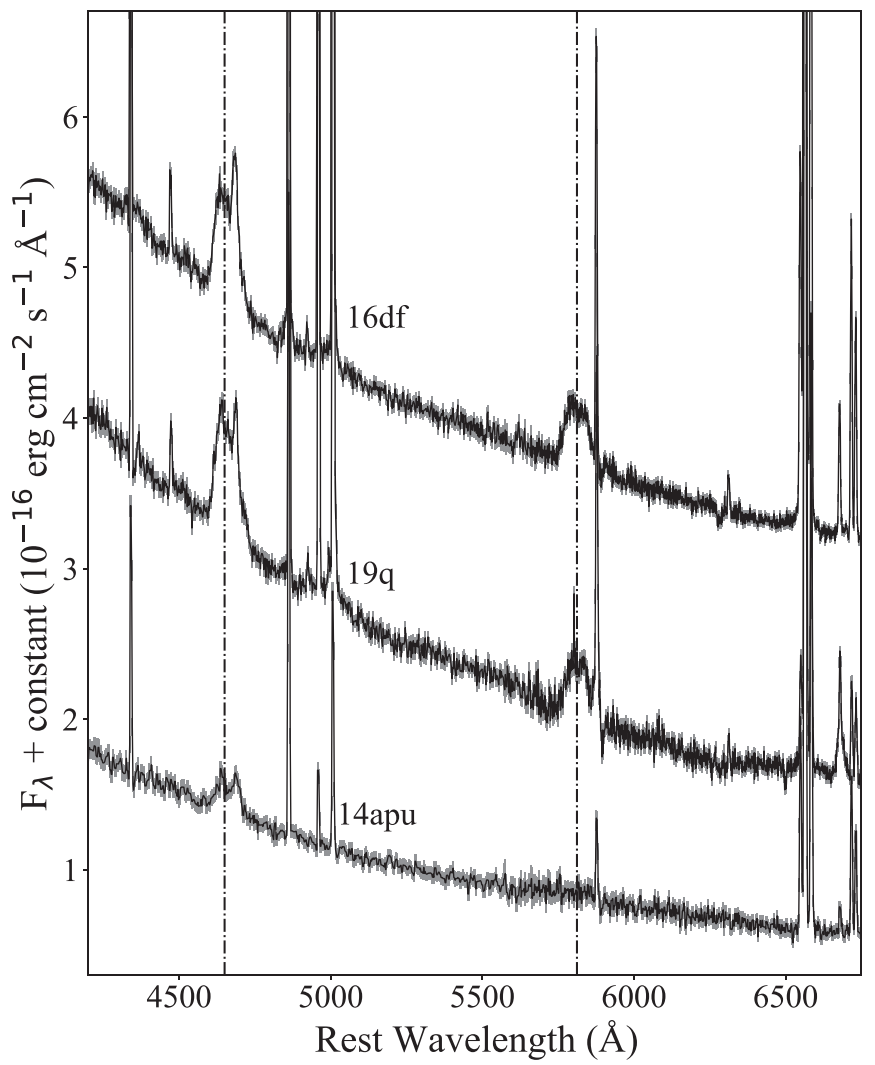

Figure 3. Reduced and flux-calibrated Keck I/LRIS spectra of SPIRITS 16df, $19 \mathrm{q}$, and 14apu overlaid with dashed lines corresponding to the wavelengths of C IV $\lambda 5801-12$ and the C III-IV $\lambda 4650 / \mathrm{He}$ II $\lambda 4686$ blend. Narrow emission features are likely associated with the underlying or nearby $\mathrm{H}$ II regions. The spectrum of SPIRITS $16 \mathrm{df}$ is shown with a continuum offset of $2 \times 10^{-16}$ $\operatorname{erg~cm}{ }^{-1} \mathrm{~s}^{-1} \AA^{-1}$.

package Spextool developed by Cushing et al. (2004), updated by M. Cushing specifically for NIRES. Telluric corrections and flux calibrations were performed with the standard-star observations using the method developed by Vacca et al. (2003)

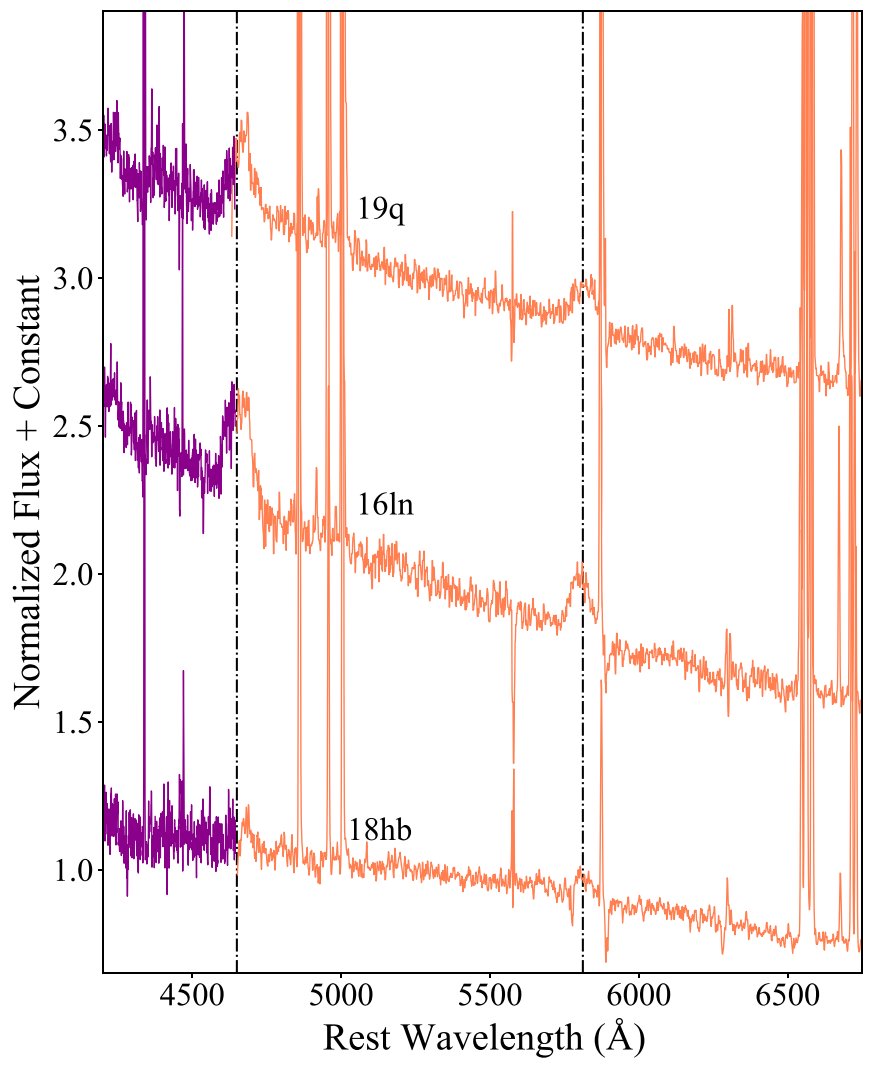

Figure 4. Reduced HET/LRS2 spectra of SPIRITS 19q, 16ln, and 18hb showing the flux normalized to the peak emission of the C III-IV $\lambda 4650 / \mathrm{He}$ II $\lambda 4686$ blend and offset for clarity. The overlaid dashed lines correspond to the wavelengths of C IV $\lambda 5801-12$ and the C III-IV $\lambda 4650 / \mathrm{He}$ II $\lambda 4686$ blend. Narrow emission features are likely associated with the underlying or nearby $\mathrm{H}$ II regions.

implemented with the IDL tools XTELLCOR or XTELLCOR_GENERAL developed by Cushing et al. (2004) as part of Spextool. The reduced spectrum normalized to the $J$-band $(1.1-1.3 \mu \mathrm{m})$ continuum flux is shown in Figure 5 with zoomed 

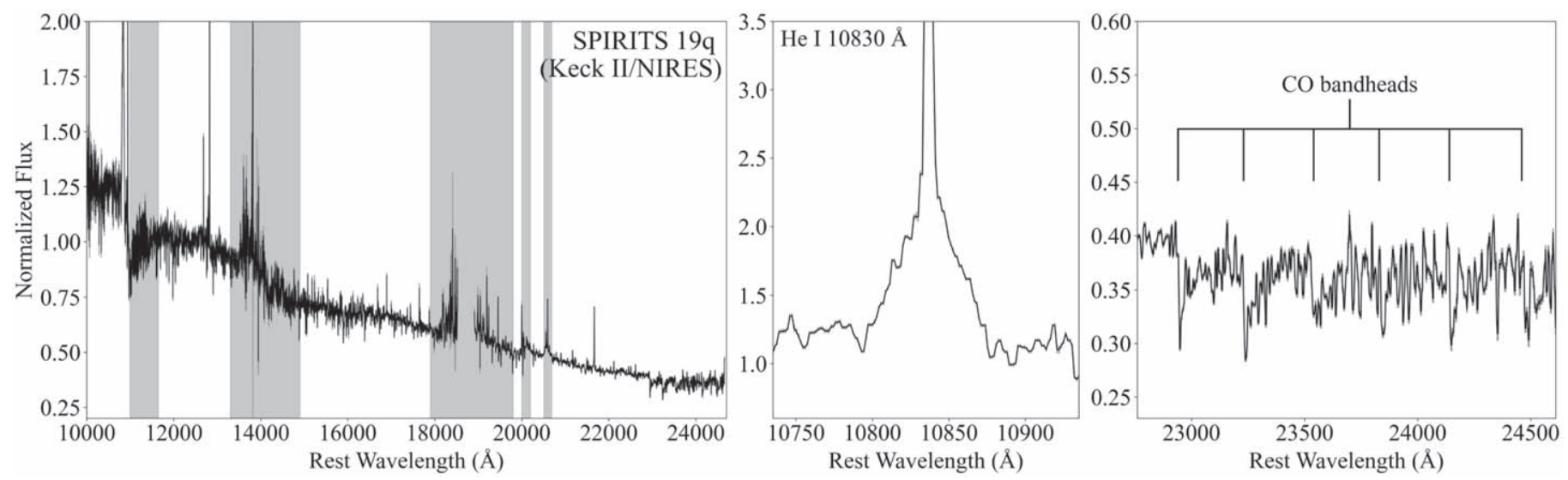

Figure 5. Reduced Keck II/NIRES spectrum of SPIRITS 19q normalized to the $J$-band continuum emission and overlaid with telluric absorption bands (in gray). Zoomed panels of the normalized spectrum on the $10830 \AA$ A He I feature and CO bandheads are shown to the right.

panels on the broadened $10830 \AA$ He I line and molecular CO bands in absorption.

\subsection{Near-IR Imaging with P200/WIRC}

Near-IR imaging of SPIRITS 14bqe in IC 1613 were taken as part of the ground-based near-IR follow-up campaign for SPIRITS using the Wide Field Infrared Camera (WIRC; Wilson et al. 2003) on the 200-inch telescope at Palomar Observatory (P200). Images of IC 1613 were obtained in the $J$ - and $K s$-band filters on WIRC on 2016 October 11. The data were sky subtracted and then calibrated using 2MASS stars in the field of view. The measured $J$ and $K s$ magnitude (in Vega) of SPIRITS 14bqe was $J=18.94 \pm 0.04$ and $K_{s}=17.90 \pm 0.06$. The $K s$-band image of SPIRITS 14bqe is shown in Figure 2 overlaid with its $2 \sigma(0 . " 6)$ positional uncertainty and the 2 "! 4 aperture used for the Spitzer/IRAC photometry.

\section{Results and Analysis}

\subsection{Identifying Dust Formation from Spitzer/IRAC Mid-IR Colors}

Mid-IR photometry is valuable for distinguishing hot dust $\left(T_{\mathrm{d}} \sim 800 \mathrm{~K}\right)$ from the free-free emission by the ionized stellar winds of WC stars (Williams 2019). Here, we demonstrate how the Spitzer/IRAC Ch1 and Ch2 color can be used to identify an IR excess around WC stars consistent with thermal emission from circumstellar dust.

The Spitzer Ch1 and Ch2 color ([3.6] - [4.5]) corresponding to measured $\mathrm{Ch} 1$ and $\mathrm{Ch} 2$ fluxes of $F_{\nu, \mathrm{Ch} 1}$ and $F_{\nu, \mathrm{Ch} 2}$ can be expressed as

$$
[3.6]-[4.5]=2.5 \log \left(\frac{F_{\nu, \mathrm{Ch} 2}}{F_{\nu, \mathrm{Ch} 1}}\right)+2.5 \log \left(\frac{F_{\nu 0, \mathrm{Ch} 1}}{F_{\nu 0, \mathrm{Ch} 2}}\right)
$$

where $F_{\nu 0, \mathrm{Ch} 1}$ and $F_{\nu 0, \mathrm{Ch} 2}$ are the zero-magnitude fluxes at Ch1 and $\mathrm{Ch} 2$, respectively. ${ }^{14}$ Free-free emission from ionized dense winds dominates the IR emission from dust-free WC stars and can be approximated as a power law: $F_{\nu}^{f f} \propto \nu^{0.96}$ (Morris et al. 1993). Based on this free-free emission power law, the Spitzer [3.6] - [4.5] color from a dust-free WC star wind can

\footnotetext{
${ }^{14} F_{\nu 0, \mathrm{Ch} 1}=280.9 \mathrm{Jy}$ and $F_{\nu 0, \mathrm{Ch} 1}=179.7 \mathrm{Jy}$.
}

be approximated as

$$
([3.6]-[4.5])_{f f} \approx 0.24 \text {. }
$$

This is consistent with the observed mid-IR colors of dust-free WC stars in the LMC (Bonanos et al. 2009; Williams 2019).

Optically thin, mid-IR thermal dust emission at a temperature $T_{\mathrm{d}}$ can be expressed as a modified blackbody with a power law: $F_{\nu}^{\mathrm{d}} \propto B_{\nu}\left(T_{d}\right) \nu^{\beta}$, where $\beta$ is the power-law emissivity index and $B_{\nu}$ is the Planck function. Because the circumstellar dust around WC stars is believed to be carbon rich (Cherchneff et al. 2000), an emissivity index consistent with amorphous carbon dust is adopted: $\beta=1.2$ (Zubko et al. 1996). Note that dust-forming WC stars do not exhibit prominent mid-IR CO emission (van der Hucht et al. 1996) and thus we do not expect any contamination from these features in the observed [4.5] photometry. From Equation (1), the predicted Spitzer [3.6] - [4.5] for thermal dust emission at a temperature $T_{\mathrm{d}} \lesssim 800 \mathrm{~K}$ can be approximated as

$$
([3.6]-[4.5])_{\mathrm{d}} \gtrsim 0.57,
$$

which is redder than the dust-free WC stars dominated by freefree emission. We therefore use Equation (3) as a criterion for identifying thermal emission from circumstellar dust.

Due to the spatial resolution of Spitzer/IRAC (FWHM $1 . " 7)$ and the location of the dust-forming WC candidates in crowded and/or dusty regions, the mid-IR photometry is likely contaminated by emission from nearby stars or nebulosity. The absolute mid-IR magnitudes from the aperture photometry of the WC candidates (Table 2) indeed exceed the absolute magnitudes of individual WC stars in the LMC measured by Spitzer/IRAC in the Surveying the Agents of the Galaxy's Evolution (SAGE) program (Meixner et al. 2006; Bonanos et al. 2009). In order to remove the contaminating emission from the WC candidates with excessive mid-IR brightness, we use the multiepoch Spitzer coverage to characterize and subtract the quiescent, nonvariable emission. Table 3 provides the nearby $\mathrm{H}$ II or star-forming regions and summarizes the properties of the dust-forming WC candidates.

\subsection{Episodic Dust Formation from the WC Binary SPIRITS16ln/N604-WRXc in M33}

SPIRITS $16 \mathrm{ln}$ is located in the massive H II region NGC 604 within the nearby galaxy M33 at a distance of $840 \mathrm{kpc}$ 
Table 3

Dust-forming WC Candidate Properties

\begin{tabular}{|c|c|c|c|c|c|c|c|}
\hline ID & Nearby H II/SF Region & Ref. & $Z / Z_{\odot}$ & Ref. & WR Class & Var. Type & Period $P(\mathrm{yr})$ \\
\hline $16 \ln$ & NGC 604 & Ga19 & 0.65 & Vi88, Ga19 & WC4 & Episodic & $8.8>P>2.2$ \\
\hline $19 q$ & NGC 2403 VS 51 & VS65 & 0.6 & Mo10 & WC4 & Episodic & $P \gtrsim 12$ \\
\hline $16 \mathrm{df}$ & NGC 2403 VS 41 & VS65 & 0.5 & Mo10 & WC4 & Irregular & $?$ \\
\hline $14 \mathrm{apu}$ & NGC5457 + $166.4+86.3$ & Cr16 & 0.5 & Cr16 & WCE? & Irregular & $?$ \\
\hline $18 \mathrm{hb}$ & NGC 6946-26 & Kh14 & 0.5 & Kh14 & WCE & Episodic? & $?$ \\
\hline 14bqe & IC 1613 Reg 155 & $\mathrm{Me09}$ & 0.16 & $\mathrm{Br} 07$ & $?$ & Episodic & $P \sim 8.5$ \\
\hline
\end{tabular}

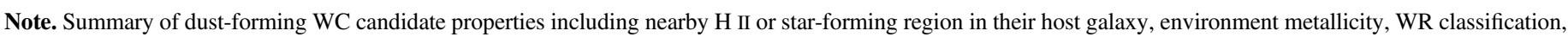

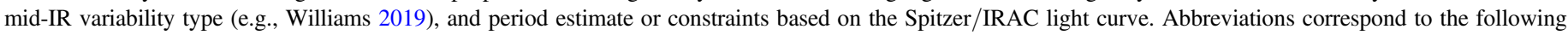

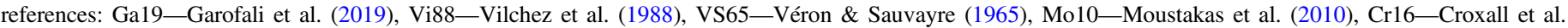
(2016), Kh14-Khramtsova et al. (2014), Me09-Melena et al. (2009), and Br07-Bresolin et al. (2007).

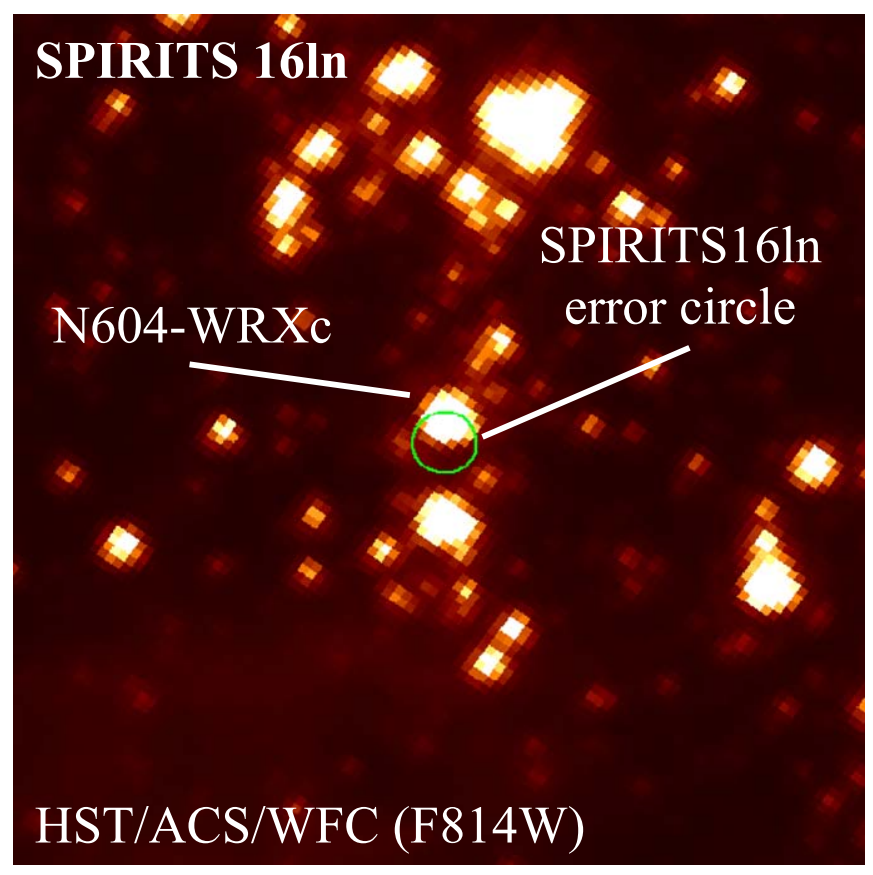

Figure 6. HST/ACS/WFC F814W image of SPIRITS 16ln/N604-WRXc taken on 2017 August 4 (Garofali et al. 2019) overlaid with the $1 \sigma\left(00^{\prime \prime} 165\right)$ positional error circle of SPIRITS $16 \mathrm{ln}$. The length and width of the image cutout is 2 ! $4 \times 2$." 4 . North is up and east is to the left.

$(\mu=24.63$; Gieren et al. 2013). NGC 604 exhibits a subsolar metallicity $Z=0.65 Z_{\odot}$ (Vilchez et al. 1988; Magrini et al. 2007; Garofali et al. 2019) comparable to that of the LMC. The Spitzer-derived position of SPIRITS $16 \mathrm{ln}$ is consistent within 0 " 13 of the $\mathrm{WC} 4+\mathrm{O}$ candidate colliding-wind binary system N604-WRXc (Garofali et al. 2019). The early-type WC emission features shown in the HET/LRS2 spectra of SPIRITS 16ln (Figure 4) are notably consistent with the spectra of N604-WRXc presented by Garofali et al. (2019). In Figure 6 we show the $1 \sigma$ positional error circle of SPIRITS 16ln after an astrometric alignment between the Spitzerimage and the Hubble Space Telescope (HST) ACS/WFC image of N604WRXc taken with the F814W filter on 2017 August 4 (Garofali et al. 2019). The position of SPIRITS $16 \mathrm{ln}$ is indeed consistent with N604-WRXc, and we therefore claim that SPIRITS 16ln is associated with N604-WRXc.

SPIRITS 16ln was observed by Spitzer in quiescence between 2003 December 28-2006 February 4 (MJD 5300153770). Between 2015 March 30-2016 April 13 (MJD 5711157491), SPIRITS 16ln was observed to be fading from a
mid-IR outburst that should have occurred between 2006 February and 2015 March. Figure 7 shows the mid-IR light curve of SPIRITS $16 \mathrm{ln}$ overplotted with the unabsorbed X-ray fluxes of N604-WRXc reported by Garofali et al. (2019). During quiescence the median 3.6 and $4.5 \mu \mathrm{m}$ emission was $F_{3.6}=$ $0.632 \pm 0.030 \mathrm{mJy}\left(M_{[3.6]}=-10.50 \pm 0.05\right)$ and $F_{4.5}=0.597 \pm$ $0.026 \mathrm{mJy} \quad\left(M_{[4.5]}=-10.92 \pm 0.05\right)$, respectively. Unfortunately, the X-ray flux peak from N604-WRXc was observed in 2006 June 9, about four months after the final Spitzer observation of SPIRITS 16ln in quiescence. It is therefore unclear if the mid-IR and X-ray emission peaks are correlated.

The peak mid-IR emission from SPIRITS 16ln was observed on 2015 March 30 (MJD 57111), where the measured fluxes were $F_{3.6}=0.973 \pm 0.029 \mathrm{mJy}\left(M_{[3.6]}=-10.97 \pm 0.03\right)$ and $F_{4.5}=0.979 \pm 0.026 \mathrm{mJy}\left(M_{[4.5]}=-11.46 \pm 0.03\right)$. Given the rapid fading following the peaks in both the mid-IR and X-ray emission from SPIRITS 16ln/N604-WRXc, it is difficult to constrain a possible recurrence period of the outbursts. However, a lower limit of $\gtrsim 2.2 \mathrm{yr}$ can be estimated from the time span of the Spitzer observations where SPIRITS 16ln was in quiescence. Semicontemporaneous mid-IR and X-ray flux peaks are associated with periastron passage in dust-forming colliding-wind binaries (e.g., Williams et al. 1990). Under the assumption that the mid-IR and X-ray peaks are indeed correlated, the upper limit of the outburst period is $\lesssim 8.8 \mathrm{yr}$ (Figure 7).

The Spitzer/IRAC [3.6] - [4.5] color and absolute [3.6] magnitude of SPIRITS $16 \mathrm{ln}$ during quiescence and at its emission peak is shown in the mid-IR CMD in Figure 8. Because the quiescent emission contributes a significant fraction of the observed mid-IR flux at the outburst peak, Figure 8 also shows the color and magnitude at the outburst peak where the median emission from the quiescent phase has been subtracted. The subtracted absolute [3.6] magnitude at peak emission is $M_{[3.6]}=-9.8$ and the mid-IR color is [3.6] $-[4.5] \sim 0.6$, which is consistent with hot dust emission (Equation (3)). The redward shift in the mid-IR colors during outburst is also indicative of dust formation. The subtracted [3.6] - [4.5] color and absolute [3.6] magnitude exhibited by SPIRITS $16 \mathrm{ln}$ during its observed peak is also consistent with the variable dust-forming WC4 system HD 36402 around its mid-IR peak (Bonanos et al. 2009; Williams et al. 2013).

Based on our analysis and the previously reported X-ray variability (Garofali et al. 2019), we claim that SPIRITS 16ln/ N604-WRXc is an episodic dust-forming colliding-wind binary hosting a WC4 star. We attribute the rapid decrease in the 

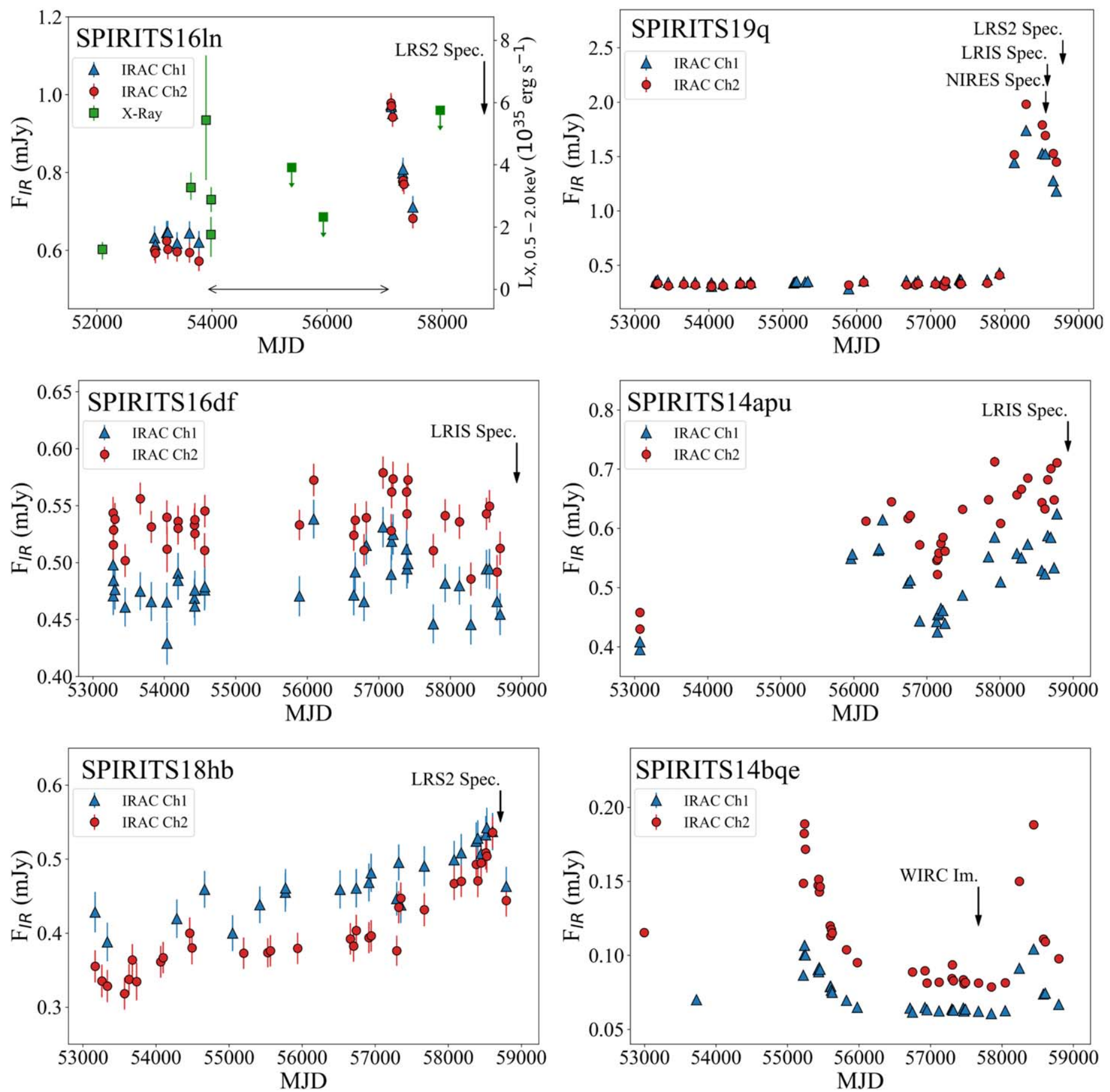

Figure 7. Spitzer/IRAC light curves at Channel $1(3.6 \mu \mathrm{m})$ and $2(4.5 \mu \mathrm{m})$ of the six dust-forming WC candidates identified in SPIRITS. The Spitzer light curve of SPIRITS $16 \mathrm{ln}$ /N604-WRXc is overlaid with the unabsorbed X-ray luminosity $(0.5-2.0 \mathrm{keV})$ measurements and upper limits from Chandra and XMM-Newton of N604-WRXc by Garofali et al. (2019); the horizontal double-sided arrow corresponds to the length of time ( $\sim 8.8$ yr) between the X-ray and mid-IR emission peaks. Dates of follow-up observations with LRIS, LRS2, and/or WIRC are indicated on the light curves.

mid-IR emission during outburst to the fading and cooling of dust as it disperses after a brief formation period during periastron passage in a highly eccentric binary orbit. This process is demonstrated in several Galactic dust-forming colliding-wind systems such as WR 19 and WR 140 (Williams et al. 2009a, 2009b). The interpretation of a binary with a high orbital eccentricity in SPIRITS 16ln is also supported by the X-ray variability reported by Garofali et al. (2019) because the $\mathrm{X}$-ray luminosity in colliding-wind binaries is highly dependent on orbital separation (e.g., Stevens et al. 1992). SPIRITS
$16 \ln / \mathrm{N} 604-W R X c$ is therefore the first extragalactic dustforming colliding-wind WC binary identified beyond the LMC.

\subsection{Candidate Dust-forming WC Stars in NGC 2403: SPIRITS $19 q$ and SPIRITS $16 d f$}

\subsubsection{Host H II Region Environments}

SPIRITS $19 \mathrm{q}$ and $16 \mathrm{df}$ are located in dusty and bright $\mathrm{H}$ II regions within the spiral galaxy NGC 2403 at a distance of 


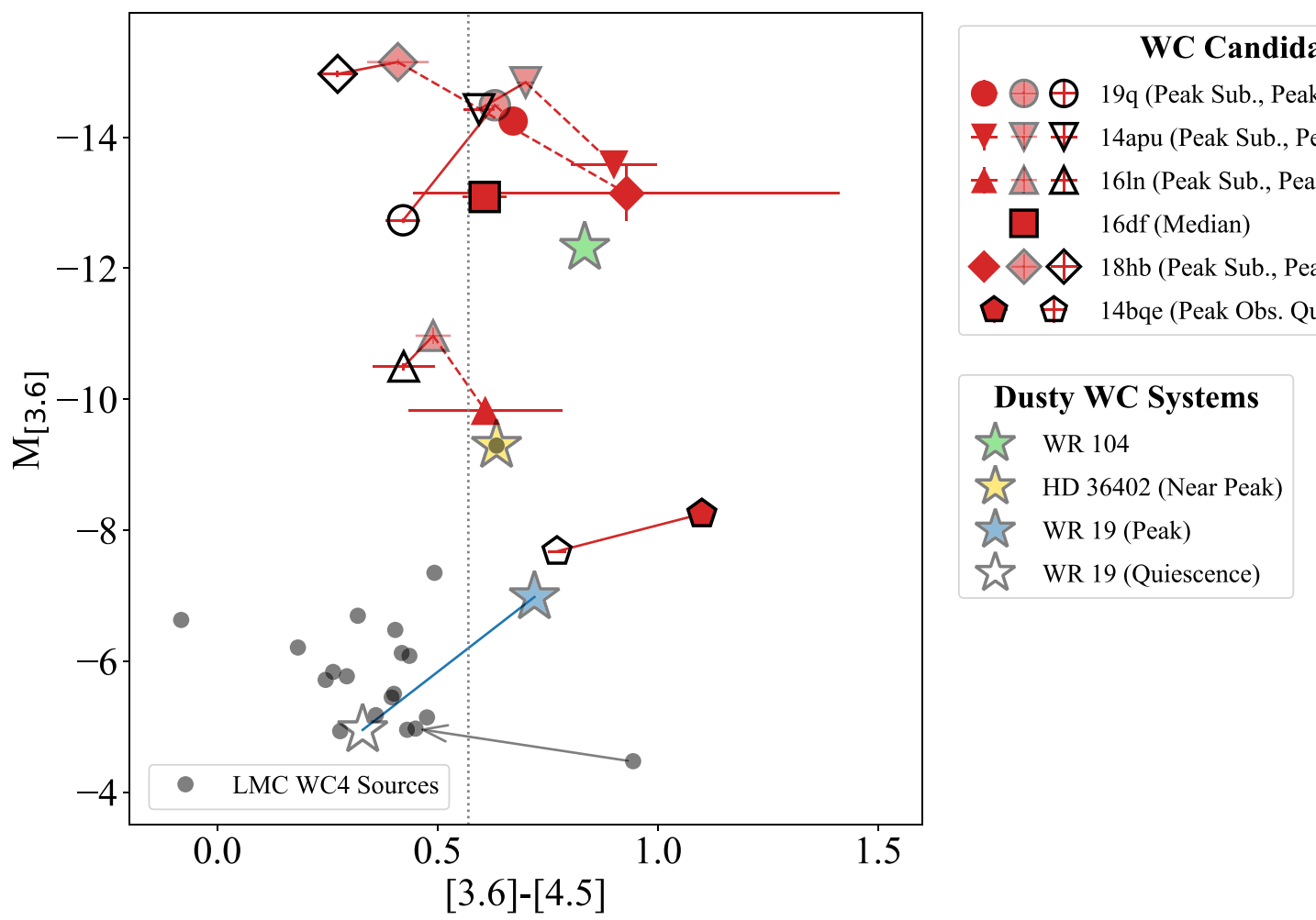

Figure 8. Mid-IR color-magnitude diagram of the six dust-forming WC candidates, LMC WC4 sources (Bonanos et al. 2009), and three known dusty WC systems: WR 104, HD 36402 near its IR peak, and WR 19 at its IR peak. Sources to the right of the vertical dotted line exhibit colors consistent with thermal dust emission at a temperature $T_{\mathrm{d}} \lesssim 800 \mathrm{~K}([3.6]-[4.5] \gtrsim 0.57$, Equation (1)). Unfilled markers correspond to source colors and magnitudes at quiescence or IR minimum. For SPIRITS 19q, 14apu, 16ln, 18hb, 14bqe and WR 19, the filled markers with borders correspond to observed magnitudes and colors at their IR peaks, whereas borderless markers indicate where the quiescent and/or underlying background emission has been subtracted. The values of [3.6] - [4.5] and $M_{[3.6]}$ for WR 104 were derived from IR spectroscopy taken by the Short Wavelength Spectrometer (SWS) on the Infrared Space Observatory (ISO; van der Hucht et al. 1996). IR peak and quiescent mid-IR colors and absolute magnitudes of WR 19 were obtained from $L^{\prime}$ - and $M$-band images taken by the IRAC1 camera on the ESO $2.2 \mathrm{~m}$ telescope in 1998 March (Veen et al. 1998) and from NEOWISE-R W1 and W2 photometry (Williams 2019), respectively. Distances toward WR 19 and WR 104 were adopted from Rate \& Crowther (2020). The revised Spitzer [3.6] - [4.5] and $M_{[3.6]}$ of the apparent red LMC WC4 outlier, HD 32125, is indicated by the arrow (See Section 3.5).

3.2 Mpc ( $\mu=27.51$; Radburn-Smith et al. 2011). Their locations within NGC 2403 are shown in Figure 1 and their Spitzer mid-IR light curves are presented in Figure 7. SPIRITS 19q and 16df are coincident with the VS 51 and VS 41 H II regions (Véron \& Sauvayre 1965), respectively. VS 51 is located at a deprojected distance of $3.5 \mathrm{kpc}$ from the nuclear core of NGC 2403 and VS 41 is located slightly closer to the core at a deprojected distance of $2.6 \mathrm{kpc}$ (Garnett et al. 1997).

VS 51 and VS 41 exhibit oxygen abundances of $12+$ $\log (\mathrm{O} / \mathrm{H})_{\mathrm{VS} 51}=8.43 \pm 0.13$ and $12+\log (\mathrm{O} / \mathrm{H})_{\mathrm{Vs} 41}=8.49 \pm$ $0.04^{15}$ (Moustakas et al. 2010), both of which are lower than the solar oxygen abundance of $12+\log (\mathrm{O} / \mathrm{H})_{\odot}=8.69 \pm 0.05$ (Asplund et al. 2009). This implies that VS 51 and VS 41 host subsolar metallicity environments where $Z_{\mathrm{VS} 51} \sim 0.6 Z_{\odot}$ and $Z_{\mathrm{VS} 41} \sim 0.5 Z_{\odot}$, which are similar to the LMC and NGC 604 . Both regions also exhibit high $0.5-2.0 \mathrm{keV}$ X-ray luminosities of $L_{\mathrm{X}}>10^{35} \mathrm{erg} \mathrm{s}^{-1}$ that are most likely powered by supernovae and/or stellar winds from massive stars (Yukita et al. 2010). Optical spectroscopy of VS 41 (as NGC 2403-V) obtained by Drissen et al. (1999) reveals WR features consistent with emission from the CIV $\lambda 5801-12$ and the C III-IV $\lambda 4650 /$ He II $\lambda 4686$ blend. Drissen et al. (1999) notably identify six WR stars in VS 41, four of which are confined to

\footnotetext{
15 These are the oxygen abundances derived by Moustakas et al. (2010) from the Pilyugin \& Thuan (2005) calibration, which is applicable to high-excitation star-forming regions such as VS 51 and VS 41.
}

the $\sim 1$ " 5 around the bright central core of the $\mathrm{H}$ II region that also contains a compact star cluster.

\subsubsection{SPIRITS $19 q$}

Rising mid-IR emission from SPIRITS $19 \mathrm{q}$ was captured by Spitzer on 2017 June 26 (MJD 57930), which peaked almost exactly a year later on 2018 June 20 (MJD 58289), where it exhibited absolute mid-IR magnitudes of $M_{[3.6]}=$ $-14.49 \pm 0.01$ and $M_{[4.5]}=-15.12 \pm 0.01$ (Figure 7, Table 2). The final Spitzer observations of SPIRITS $19 \mathrm{q}$ were obtained on 2019 August 2 (MJD 58697) and show that its mid-IR emission has faded at a rate of $\sim 0.4 \mathrm{mag} \mathrm{yr}^{-1}$ at $3.6 \mu \mathrm{m}$ and $\sim 0.3 \mathrm{mag}$ $\mathrm{yr}^{-1}$ at $4.5 \mu \mathrm{m}$ after the peak.

SPIRITS $19 \mathrm{q}$ was in a quiescent phase for at least $\sim 12$ years between 2004 October 7-2017 January 10 (MJD 53285-57764) before the 2018 outburst. During quiescence, the median midIR emission from SPIRITS 19q was $F_{3.6}=0.345 \pm 0.009 \mathrm{mJy}$ and $F_{4.5}=0.326 \pm 0.008 \mathrm{mJy} \quad\left(M_{[3.6]}=-12.73 \pm 0.03\right.$ and $\left.M_{[4.5]}=-13.16 \pm 0.03\right)$. The bright mid-IR absolute magnitudes during quiescence $\left(M_{[3.6]}<-12.5\right.$ and $\left.M_{[4.5]}=-13.0\right)$ suggest that SPIRITS $19 \mathrm{q}$ is located within an $\mathrm{H}$ II region or star cluster (e.g., Lau et al. 2019), which is also supported by Spitzerimaging observations that reveal slightly extended emission from 19q (Figure 1).

Figure 8 shows the absolute [3.6] magnitude and [3.6][4.5] color of SPIRITS 19q during quiescence and at its mid-IR 


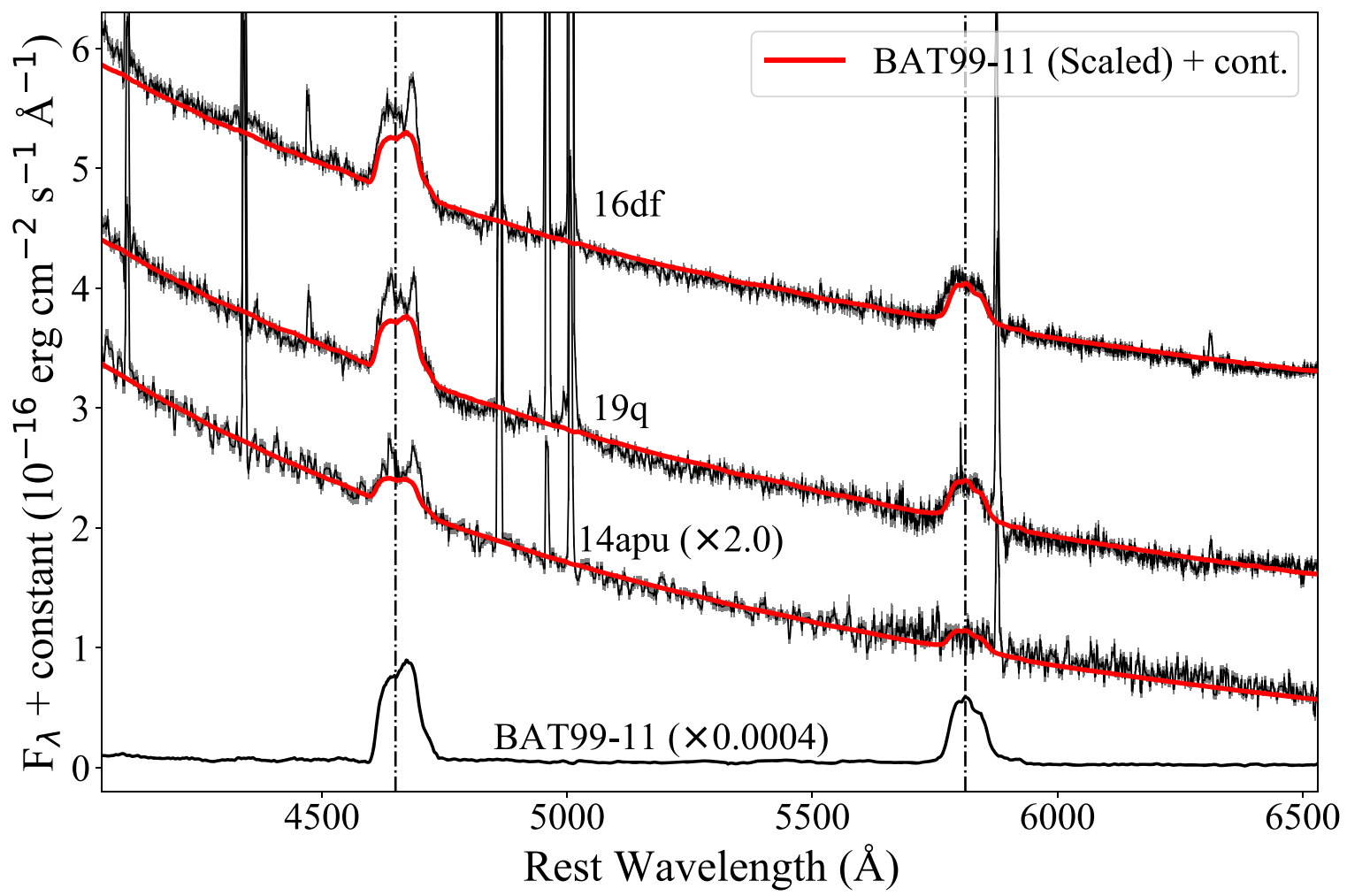

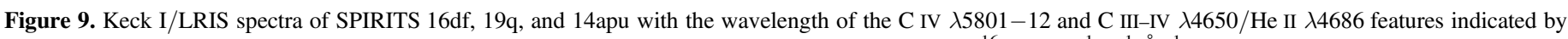

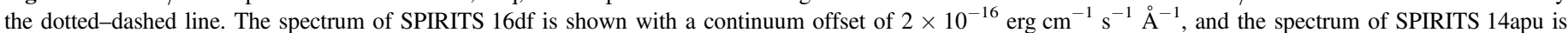

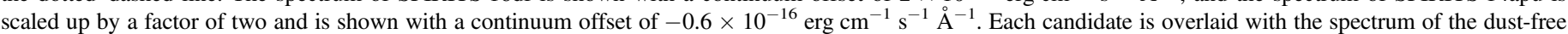

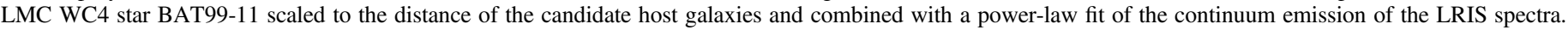

peak with and without the quiescent emission subtracted. SPIRITS $19 \mathrm{q}$ demonstrates a clear redward shift in its [3.6] - [4.5] color from quiescence to its outburst phase, which suggests that dust formation is associated with the outburst. The quiescence-subtracted mid-IR color at its emission peak is [3.6] $-[4.5]=0.67 \pm 0.01$, consistent with thermal dust emission as well as the mid-IR colors exhibited by SPIRITS 16ln during its outburst and HD 36402. Figure 8 also shows the observed mid-IR color and magnitude of WR 104, a well-studied persistent dust-forming WC9+OB binary in our Galaxy that is one of the most IR-luminous dust-forming WC systems known (Tuthill et al. 1999; Soulain et al. 2018; Lau et al. 2020a). Interestingly, the quiescence-subtracted absolute [3.6] magnitude of SPIRITS $19 \mathrm{q}$ during outburst, $M_{[3.6]}=$ $-14.25 \pm 0.01$, exceeds that of WR 104 by almost two magnitudes. This suggests substantial dust formation from SPIRITS $19 \mathrm{q}$ during its outburst.

Optical Keck/LRIS spectra of SPIRITS 19q were obtained within a year of the 2018 outburst peak. In addition to narrow emission lines likely associated with the nearby $\mathrm{H}$ II region, the spectrum reveals clear detections of broad emission features from C IV $\lambda 5801-12$ and the C III-IV $\lambda 4650 / \mathrm{He}$ II $\lambda 4686$ blend (Figure 3 ). These features are consistent with a WC star, and the absence of the lower excitation $5696 \AA \mathrm{C}$ III line suggests an early WC subtype (Crowther et al. 1998). SPIRITS $19 q$ notably exhibits the same broad WC emission features as the WC4 star in N604-WRXc (Figure 4; Garofali et al. 2019). The observed continuum emission at $\lambda=5500 \AA$ in the LRIS spectrum of SPIRITS $19 \mathrm{q}$ corresponds to a $V$-band magnitude of $V \approx 18.0$ and an absolute magnitude of $M_{V} \approx-9.5$. The absolute $V$-band magnitude exceeds that of the brightest supergiants in the LMC $\left(M_{V} \approx-8.5\right.$; Bonanos et al. 2009), which indicates that SPIRITS $19 q$ is most likely associated with a luminous stellar cluster.

In Figure 9 the LRIS spectrum of SPIRITS $19 q$ is overlaid with the spectrum of the dust-free LMC WC4 star BAT99-11 (=Brey 10, HD 32402; Torres \& Massey 1987; Neugent et al. 2018) that has been scaled to the distance of NGC $2403^{16}$ and combined with a power-law fit of the continuum emission from the $19 q$ spectrum. The amplitude and profile of the WC features in the distance-scaled and continuum-offset spectrum of BAT99-11 show a close agreement with the features from SPIRITS $19 q$. We therefore claim that SPIRITS $19 q$ hosts a WC4 star.

The near-IR spectrum of SPIRITS $19 q$ in Figure 5 shows a narrow 10830 A He I feature combined with a broad component. This broad component appears consistent with the profile of the $10830 \AA$ He I feature exhibited by Galactic WR stars (Howarth \& Schmutz 1992; Groh et al. 2007). The detection of $\mathrm{CO}$ absorption bands as well as a possible $\mathrm{CN}$ absorption feature at around $1.1 \mu \mathrm{m}$ suggests that cooler supergiants (Rayner et al. 2009) are also present in the VS 51 $\mathrm{H}$ II region.

Because SPIRITS $19 q$ is likely associated with a star cluster in the VS $51 \mathrm{H}$ II region, we must consider other possible origins of the 2018 outburst beyond episodic dust formation from a WC4 binary system. We can rule out several possibilities based on its optical and near-IR spectra. Given the mid-IR magnitude and color of SPIRITS 19q, one of the most plausible origins of the outburst would be a highly

\footnotetext{
${ }^{16}$ Assuming an LMC distance of $50 \mathrm{kpc}$.
} 
obscured core-collapse SN (Jencson et al. 2019). The optical and near-IR spectra, however, show no clear evidence of broadened ( $\gtrsim 2000 \mathrm{~km} \mathrm{~s}^{-1}$ ) hydrogen emission lines that are characteristic of core-collapse SNe. SPIRITS $19 q$ is therefore unlikely associated with an obscured supernova. The lack of broad hydrogen emission features also suggests SPIRITS $19 \mathrm{q}$ is not associated with a giant eruption from a luminous blue variable (LBV; e.g., Smith 2014). Other possible origins include a massive stellar merger like NGC 4490 OT2011 (Smith et al. 2016) or an intermediate-luminosity red transient (ILRT) like NGC 300 OT2008 (Bond et al. 2009), but these events should be much rarer than a dust formation episode in a WC binary. We therefore consider SPIRITS $19 \mathrm{q}$ as a candidate episodic dust-forming WC system that exhibits efficient dust formation.

\subsubsection{SPIRITS $16 d f$}

SPIRITS 16df exhibits irregular and low-amplitude mid-IR variability in the Spitzer observations that span 2004 October 7-2019 August 2 (MJD 53285-58697; Figure 7). The median mid-IR emission from SPIRITS 16df was $F_{3.6}=0.479 \pm$ $0.017 \mathrm{mJy}$ and $F_{4.5}=0.536 \pm 0.015 \mathrm{mJy}\left(M_{[3.6]}=-13.09 \pm\right.$ 0.04 and $\left.M_{[4.5]}=-13.70 \pm 0.03\right)$, and the maximum amplitude of the observed variability from the median mid-IR emission was $\sim 10 \%$ ( $\sim 0.1 \mathrm{mag})$. The amplitude and timing of the variability is consistent at both 3.6 and $4.5 \mu \mathrm{m}$. Extended emission surrounding SPIRITS 16df in the nonsubtracted Spitzer image (Figure 1) suggests that it is associated with an $\mathrm{H}$ II region (i.e., VS 41).

Optical spectroscopy of SPIRITS 16df shown in Figure 3 was obtained on 2020 March 23 (MJD 58931) with Keck/ LRIS and shows the C IV $\lambda 5801-12$ and the C III-IV $\lambda 4650 /$ He II $\lambda 4686$ emission features. This LRIS spectrum of SPIRITS 16df is consistent with the optical spectrum of VS 41 presented by Drissen et al. (1999). The observed continuum emission from the LRIS spectrum of SPIRITS 16df at $\lambda=5500 \AA$ corresponds to a $V$-band magnitude of $V \approx 18.2$ and an absolute magnitude of $M_{V} \approx-9.3$. Similar to the LRIS spectrum of SPIRITS $19 \mathrm{q}$, the bright absolute $V$-band magnitude from the SPIRITS 16df spectrum indicates that it is associated with a luminous stellar cluster. HST/WFPC2 imaging of the SPIRITS 16df host H II region by Drissen et al. (1999) indeed identified a compact cluster adjacent to the WR stars in VS 41.

The spectrum of SPIRITS 16df shows a strong resemblance to the WC features from SPIRITS $19 q$ and SPIRITS $16 \ln /$ N604-WRXc (Garofali et al. 2019). In Figure 9 the LRIS spectrum of SPIRITS 16df is overlaid with the distance-scaled spectrum of the LMC WC4 star BAT99-11 combined with a power-law fit of the continuum emission from 16df. The close agreement with the WC4 features from BAT99-11 indicates that SPIRITS 16df hosts a WC4 star.

Because the mid-IR light curve of SPIRITS 16df does not exhibit obvious quiescent and outburst states like SPIRITS $19 \mathrm{q}$ or $16 \mathrm{ln}$, the CMD in Figure 8 only shows its median absolute [3.6] magnitude and median Spitzer mid-IR color $([3.6]-[4.5]=0.61 \pm 0.05)$. This mid-IR color is consistent with emission from hot dust and is also similar to that of SPIRITS 19q, 16ln, and HD 36402 (Figure 8). However, it is difficult to determine what fraction of the emission might originate from the WC star compared to the H II region or other nearby stars. We consider SPIRITS $16 \mathrm{df}$ as a possible dust-forming WC binary, but further multiwavelength monitoring observations will be crucial to confirm this hypothesis.

3.4. Candidate Dust-forming WC Stars in Messier 101, NGC 6946, and IC 1613: SPIRITS 14apu, SPIRITS 18hb, and SPIRITS 14bqe

\subsubsection{SPIRITS 14apu}

SPIRITS 14apu was identified in the nearby spiral galaxy M101 (Figure 1) at $d=6.4 \mathrm{Mpc}(\mu=29.04$; Shappee \& Stanek 2011) and is located within 3!'7 of the H II region NGC5457+166.4 + 86.3 at a deprojected galactocentric radius of $\sim 5.8 \mathrm{kpc}$ (Croxall et al. 2016). This H II region exhibits an oxygen abundance of $12+\log (\mathrm{O} / \mathrm{H})=8.42 \pm 0.05$ (Croxall et al. 2016), which implies an LMC-like subsolar metallicity environment with $Z \sim 0.5 Z_{\odot}$. Croxall et al. (2016) also indicate the presence of WR features in optical spectroscopy of this H II region. Although SPIRITS 14apu may not be located within this H II region given the 3 !! $7(\sim 120 \mathrm{pc})$ offset, we assume that the surrounding environment of 14apu hosts a similar subsolar metallicity.

Spitzer observations of SPIRITS 14apu (Figure 7) revealed slow, irregular mid-IR variability with a prominent dip around late 2014/early 2015 (MJD 57000) followed by a gradual brightening that continued until the final observation taken on 2019 October 25 (MJD 58781). At this final epoch, SPIRITS 14apu exhibited its maximum observed emission at $3.6 \mu \mathrm{m}$ with $F_{3.6}=0.62 \pm 0.01 \mathrm{mJy}\left(M_{[3.6]}=-14.91 \pm 0.02\right)$ and $F_{4.5}=0.71 \pm 0.01 \mathrm{mJy}\left(M_{[4.5]}=-15.53 \pm 0.01\right)$. Given its bright absolute mid-IR magnitudes, SPIRITS 14apu is likely associated with a star cluster and/or dusty $\mathrm{H}$ II region.

Two observations from 2004 March 8 (MJD 53072) captured SPIRITS 14apu at its minimum observed mid-IR brightness, where the average 3.6 and $4.5 \mu \mathrm{m}$ emission was $F_{3.6}=0.40 \pm 0.01 \mathrm{mJy}\left(M_{[3.6]}=-14.43 \pm 0.03\right)$ and $F_{4.5}=$ $0.44 \pm 0.01 \mathrm{mJy} \quad\left(M_{[4.5]}=-15.02 \pm 0.02\right)$, respectively. At this minimum brightness phase, SPIRITS 14apu exhibited a mid-IR color of [3.6] $-[4.5]=0.59 \pm 0.04$ (Figure 8). It is assumed that the mid-IR emission at this observed minimum captures the nonvariable emission from the nearby $\mathrm{H}$ II region and/or cluster associated with SPIRITS 14apu. The cluster/ $\mathrm{H}$ II region-subtracted absolute [3.6] magnitude and mid-IR color of SPIRITS 14apu at its peak mid-IR brightness on 2019 October 25 is $M_{[3.6]}=-13.58 \pm 0.08$ and [3.6] $-[4.5]=$ $0.90 \pm 0.10$. Between the observed phases of minimum and maximum mid-IR emission, SPIRITS 14apu exhibited a redward shift in its mid-IR color consistent with the formation of hot dust. The absolute [3.6] magnitude of SPIRITS 14apu at its mid-IR peak was greater than WR 104 even after subtraction of the underlying cluster/H II region emission, which suggests a high dust-formation rate relative to WR 104.

Spectroscopic observations of SPIRITS 14apu were obtained with Keck/LRIS on 2020 March 23 (MJD 58931) and show the C III-IV $\lambda 4650 / \mathrm{He}$ II $\lambda 4686$ emission complex but present no significant detection of the C IV $\lambda 5801-12$ emission feature (Figure 3). The observed continuum emission at $\lambda=5500$ $\AA$ corresponds to a $V$-band magnitude of $V \approx 19.1$ and an absolute magnitude of $M_{V} \approx-10.0$. This is consistent with the interpretation that SPIRITS 14apu is associated with a luminous stellar cluster. 
Figure 9 shows the LRIS spectrum of SPIRITS 14apu overlaid with spectrum of BAT99-11, which has been scaled to the distance of M101 and combined with a power-law fit of the continuum emission from 14apu. The width of the C III-IV $\lambda 4650 / \mathrm{He}$ II $\lambda 4686$ emission complex in SPIRITS 14apu closely matches that of the distance-scaled and continuumoffset spectrum of BAT99-11. The nondetection of the C IV $\lambda 5801-12$ feature from $14 \mathrm{apu}$ is consistent with the low amplitude expected from this feature based on the spectrum of BAT99-11. We therefore consider SPIRITS 14apu to be a candidate dust-forming WC system. Although the C III-IV $\lambda 4650 / \mathrm{He}$ II $\lambda 4686$ emission complex is indicative of a WC star, it is difficult to determine a specific WC classification for SPIRITS 14apu because the C IV $\lambda 5801-12$ feature is an important feature for distinguishing WC subtypes (e.g., Crowther et al. 1998). Given the apparent link between early spectral subtypes and low metallicities (e.g., Crowther 2007), the subsolar metallicity of its surrounding environment implies that SPIRITS 14apu likely hosts an early-type WC star.

\subsubsection{SPIRITS $18 \mathrm{hb}$}

SPIRITS $18 \mathrm{hb}$ is located in a spiral arm $\sim 2$ '. 8 from the central core of the galaxy NGC 6946 at a distance $d=7.72 \mathrm{Mpc}(\mu=$ 29.44; Anand et al. 2018). An H II region complex, NGC 6946-26, is located 3!"9 from SPIRITS $18 \mathrm{hb}$ and exhibits an oxygen abundance of $12+\log (\mathrm{O} / \mathrm{H})=8.37 \pm 0.01$ (Khramtsova et al. 2014). This implies that SPIRITS $18 \mathrm{hb}$ is in an LMC-like subsolar metallicity environment with $Z \sim 0.5 Z_{\odot}$.

Spitzer/IRAC observations of SPIRITS $18 \mathrm{hb}$ span from 2004 June 10 to 2019 November 8 (MJD 53167-58795) and show relatively steady mid-IR emission from $18 \mathrm{hb}$ until 2017 November 23 (MJD 58080; Figure 7). On 2017 November 23, the emission increased rapidly for $\sim 1 \mathrm{yr}$ and had faded at both 3.6 and $4.5 \mu \mathrm{m}$ in the last observation taken in 2019 November 8. This spike in mid-IR emission appears more consistent with episodic variability observed from WC candidates such as SPIRITS $16 \mathrm{ln}$ and $19 \mathrm{q}$ as opposed to the irregular variability observed from SPIRITS 16df and 14apu (Figure 7, Table 3).

The peak $3.6 \mu \mathrm{m}$ emission from SPIRITS $18 \mathrm{hb}$ was observed on 2019 February 15 (MJD 58529) where $F_{3.6}=0.54 \pm 0.03 \mathrm{mJy}$ $\left(M_{[3.6]}=-15.15 \pm 0.05\right)$ and $F_{4.5}=0.50 \pm 0.02 \mathrm{mJy}\left(M_{[4.5]}=\right.$ $-15.56 \pm 0.05)$. The median mid-IR emission from SPIRITS $18 \mathrm{hb}$ between 2004 June-2017 November was $F_{3.6}=$ $0.46 \pm 0.02 \mathrm{mJy} \quad\left(M_{[3.6]}=-14.98 \pm 0.04\right) \quad$ and $\quad F_{4.5}=0.38 \pm$ $0.02 \mathrm{mJy}\left(M_{[4.5]}=-15.24 \pm 0.06\right)$. The bright mid-IR emission during this low-variability, quiescent phase and the extended emission around SPIRITS 18hb (Figure 1) indicates that $18 \mathrm{hb}$ is likely associated with a stellar cluster and/or dusty $\mathrm{H}$ II region.

The mid-IR CMD in Figure 8 shows the redward shift of SPIRITS $18 \mathrm{hb}$ between its steady quiescent phase, where $[3.6]-[4.5]=0.27 \pm 0.05$, and its IR peak. The absolute [3.6] magnitude and mid-IR color at its IR peak subtracted by the quiescent phase emission is $M_{[3.6]}=-13.14 \pm 0.42$ and $[3.6]-[4.5]=0.93 \pm 0.48$, respectively. The shift in the midIR color from SPIRITS $18 \mathrm{hb}$ appears consistent with dust formation, but the uncertainties are large due to nearby extended emission and the crowded surrounding environment (Figure 1). Similar to SPIRITS 19q, 16df, and 14apu, the absolute [3.6] magnitude from SPIRITS $18 \mathrm{hb}$ during its IR peak exceeds that of the heavy dust-maker WR 104, which suggests a high dust-formation rate.
Optical spectra of SPIRITS 18hb were taken on 2019 August 20 (MJD 58715) with HET/LRS2 (Figure 4) and reveal detections of the C IV $\lambda 5801-12$ feature and C III-IV $\lambda 4650 /$ He II $\lambda 4686$ emission complex. Unfortunately, the C III-IV $\lambda 4650 / \mathrm{He}$ II $\lambda 4686$ blend falls in the transition region between the UV and Orange channels of the LRS2 spectrograph, which affects its emission profile. It is also difficult to compare the LRS2 spectra of SPIRITS $18 \mathrm{hb}$ to the WC4 star BAT99-11 (e.g., Figure 9) due to the nonphotometric conditions of LRS2 spectra observations. However, the presence of these WC features and the absence of the lower excitation 5696- $\triangle \mathrm{C}$ III emission line implies that SPIRITS $18 \mathrm{hb}$ hosts an early-type WC star. We therefore consider SPIRITS $18 \mathrm{hb}$ as a candidate dust-forming early-type WC system that exhibits episodic dust formation.

\subsubsection{SPIRITS $14 b q e$}

SPIRITS 14bqe is located in the nearby dwarf galaxy IC 1613 at a distance of $724 \mathrm{kpc}(\mu=24.30$; Hatt et al. 2017). The position of SPIRITS 14bqe is consistent with the star-forming region IC 1613 Region 155 identified by Melena et al. (2009). IC 1613 hosts a low-metallicity environment where the mean oxygen abundance of massive stars and $\mathrm{H}$ II regions is $12+\log (\mathrm{O} / \mathrm{H})=7.90 \pm 0.08$ (Bresolin et al. 2007). This implies that SPIRITS 14bqe is in a low-metallicity environment with $Z \sim 0.16 Z_{\odot}$, which is the lowest metallicity of all six dust-forming WC candidates (Table 3).

Spitzer/IRAC observations of SPIRITS 14bqe between 2010 January 26 and 2019 November 8 (MJD 55222-58795) show two mid-IR outburst peaks separated by $\sim 8.5 \mathrm{yr}$. The measured mid-IR emission was consistent at both mid-IR peaks, where $F_{3.6}=0.107 \pm 0.001 \mathrm{mJy}\left(M_{[3.6]}=-8.25 \pm 0.01\right)$ and $F_{4.5}=$ $0.189 \pm 0.001 \mathrm{mJy}\left(M_{[4.5]}=-9.35 \pm 0.01\right)$ at the 2010 February 14 (MJD 55241) peak. The 2010 February mid-IR peak faded on a timescale of two years back to quiescence, where the median emission was $F_{3.6}=0.063 \pm 0.001 \mathrm{mJy} \quad\left(M_{[3.6]}=\right.$ $-7.68 \pm 0.01)$ and $F_{4.5}=0.082 \pm 0.001 \mathrm{mJy}\left(M_{[4.5]}=-8.45 \pm\right.$ $0.01)$. The absolute mid-IR magnitude exhibited by SPIRITS 14bqe during quiescence is consistent with emission from an individual stellar source as opposed to a stellar cluster or dusty $\mathrm{H}$ II region.

Near-IR imaging of SPIRITS 14bqe taken on 2016 October 11 (MJD 57672) during mid-IR quiescence (Figure 7) with WIRC show a point-like IR counterpart at the Spitzer-derived coordinates of 14bqe (Figure 2). WIRC imaging also shows a nearby near-IR point source 1 ".8 southeast of the SPIRITS 14bqe that falls within the 2!"4 aperture used for measuring Spitzer/IRAC photometry. However, Spitzer imaging shows that there is no significant mid-IR emission associated with the southeast near-IR point source. The Spitzer photometry of SPIRITS 14bqe is therefore dominated by the emission from the central near-IR point source associated with 14bqe. The WIRC photometry measured from this near-IR counterpart is $J=18.94 \pm 0.04$ and $K_{s}=$ $17.90 \pm 0.06$, which indicates absolute $J$ and $K_{s}$ magnitudes of $M_{J}=-5.36$ and $M_{K s}=-6.40$. The average absolute $K_{s}$ magnitudes of Galactic nondusty WC stars range from $M_{K s}=$ -4.3 to -5.3 , but for Galactic dusty WC9 stars, the average absolute $K_{s}$ magnitude is $M_{K s}=-6.6 \pm 0.8$ (Rate \& Crowther 2020). The near-IR brightness from SPIRITS 14bqe therefore exceeds that of nondusty WC stars, but is consistent with the brightness of dusty WC stars. 


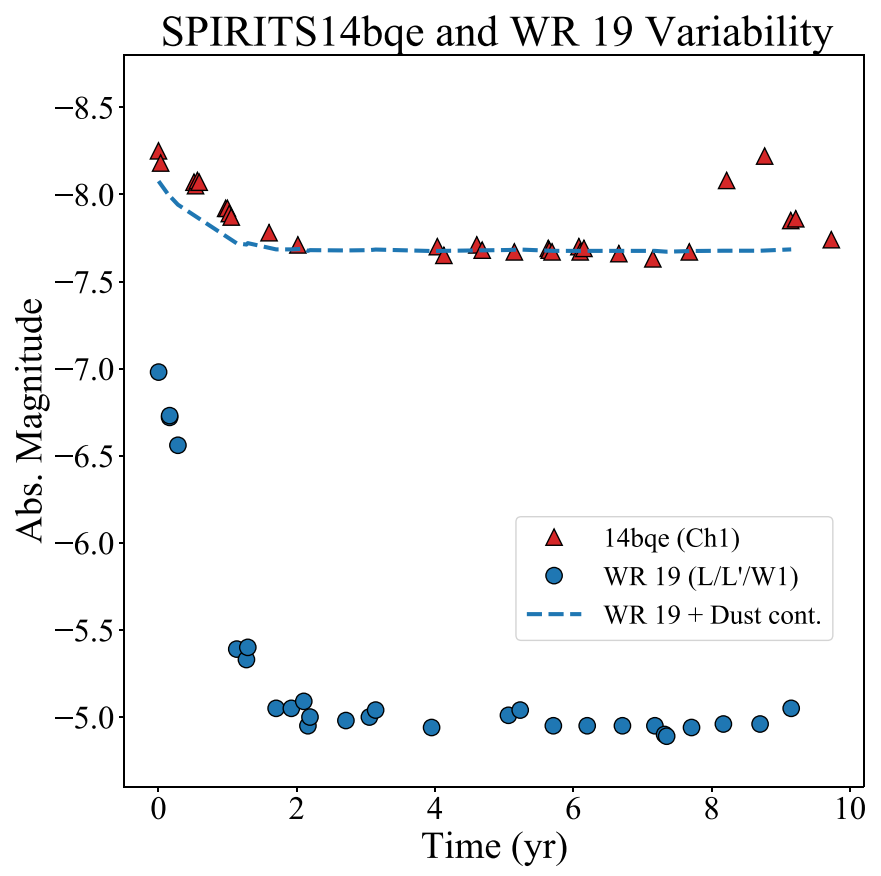

Figure 10. Mid-IR light curve of SPIRITS 14bqe between 2010 January and 2019 November (MJD 55222-58796) plotted with the phase-folded mid-IR light curve of WR 19 over its 10.1 yr orbital period (Williams et al. 1990; Veen et al. 1998; Williams 2019). The origin $t=0 \mathrm{yr}$ corresponds to the date of the maximum observed mid-IR emission from SPIRITS 14bqe and WR 19. The dashed line shows the WR 19 light curve with a constant flux offset added to match the quiescent mid-IR emission from SPIRITS 14bqe.

The mid-IR CMD in Figure 8 shows the redward shift from SPIRITS 14bqe between quiescence and its mid-IR peak, where [3.6] $-[4.5]=0.77 \pm 0.02$ and $1.10 \pm 0.01$, respectively. Interestingly, the red mid-IR color from SPIRITS 14bqe during quiescence suggests that it persistently forms dust and undergoes enhanced dust-formation episodes during the mid-IR outbursts. SPIRITS 14bqe is fainter than the peak emission from the persistent, variable dust-forming WC4 system HD 36402, but it is brighter than the peak emission from the episodic, dust-forming WC5+O9 binary system WR 19.

Figure 10 shows a comparison of the absolute mid-IR magnitude light curves from SPIRITS 14bqe and WR 19 over the 10.1 yr orbital period of WR 19 (Williams et al. 1990; Veen et al. 1998; Williams 2019). Both systems show a similar decrease in mid-IR emission that returns to quiescence over a timescale of $\lesssim 2 \mathrm{yr}$ following the outburst peak. Unlike SPIRITS 14bqe, the mid-IR emission from WR 19 is dominated by dust only during the periodic dust-formation events that correspond to periastron passage. During quiescence, midIR emission from WR 19 is largely due to the free-free emission from the stellar wind of the WC star (Williams 2019). The absence of persistent dust formation in WR 19 is apparent in the discrepancy between the absolute mid-IR magnitudes of WR 19 and SPIRITS 14bqe in their quiescent phases. In quiescence, WR 19 notably exhibits a similar mid-IR color and absolute magnitude as the dust-free WC4 stars in the LMC (Figure 8).

Figure 10 also shows the mid-IR light curve of WR 19 with a constant flux offset added to match the quiescent mid-IR emission from SPIRITS 14bqe. This approximates the emission from persistent dust formation in addition to the observed periodic dust formation in WR 19. The dust-formation peak in this modified light curve of WR 19 closely resembles the observed light curve of SPIRITS 14bqe. It is therefore plausible that SPIRITS 14bqe is a WC system exhibiting persistent and episodic dust formation. However, the lack of optical spectroscopy precludes a firm classification of SPIRITS 14bqe.

\subsection{The Apparent Red WC4 Outlier in the LMC: HD 32125}

In the mid-IR CMD (Figure 8), there are two LMC WC4 sources that exhibit red [3.6] - [4.5] colors consistent with dust emission: HD 36402 (=BAT99-38) and HD 32125 (=BAT999). HD 36402 is a known dusty WC system (Williams et al. 2013), but no evidence of circumstellar dust or mid-IR variability has been reported for HD 32125 (Williams 2019). Upon closer inspection of the SAGE IRAC Epoch 1 and Epoch 2 catalog, the apparent [3.6] magnitudes of HD 32125 present discrepant values of [3.6] $=14.05 \pm 0.09$ from Epoch 1 (2005 July) and [3.6] $=13.55 \pm 0.05$ from Epoch 2 (2005 October/ November); however, the apparent [4.5], [5.8], and [8.0] magnitudes are consistent between the two epochs. The average W1 $\left(\lambda_{c}=3.35 \mu \mathrm{m}\right)$ magnitude of HD 32125 reported by Williams (2019), who found no significant mid-IR variability from HD 32125, was $W 1=13.52 \pm 0.03$, which is consistent with the [3.6] magnitude from SAGE Epoch 2.

The [3.6] magnitude of HD 32125 in Figure 8 and from Bonanos et al. (2009) is identical to the discrepant Epoch 1 [3.6] magnitude reported in the SAGE IRAC Epoch 1 and Epoch 2 Catalog. The fainter [3.6] magnitude would account for its apparent red mid-IR color of [3.6] $-[4.5]=0.94$ in the CMD. If we instead adopt the SAGE Epoch 2 apparent [3.6] magnitude, the absolute [3.6] magnitude and mid-IR color of HD 32125 is $M_{[3.6]}=-4.97$ and [3.6] $-[4.5]=0.45$. The SAGE Epoch 2 photometry of HD 32125 is therefore consistent with the dust-free WC4 stars in the LMC (Figure 8). Given the observational evidence, we conclude that HD 32125 probably did not exhibit circumstellar dust emission, and we suggest that the fainter [3.6] measurement in SAGE Epoch 1 did not arise from any intrinsic astrophysical variability associated with HD 32125.

\section{Discussion \\ 4.1. Efficient Dust formation from SPIRITS $19 q$}

Out of all the six source in our sample, SPIRITS $19 q$ exhibited the most luminous mid-IR emission at its IR peak after subtraction of the quiescent emission (Figure 8). This suggests that SPIRITS 19q exhibits the most dust production of the sources in our sample. With the 3.6 and $4.5 \mu \mathrm{m}$ Spitzer photometry of SPIRITS $19 \mathrm{q}$, we can estimate the dust production rate during its IR outburst and compare it against other known dust-forming WC systems. Because the mid-IR dust emission from the SPIRITS $19 \mathrm{q}$ IR outburst is significantly brighter than its quiescent emission, we are able to obtain more robust estimates of the dust properties associated with the outburst.

The hot dust mass around SPIRITS $19 \mathrm{q}$ can be estimated from the 3.6 and $4.5 \mu \mathrm{m}$ flux measured by Spitzer. When we assume that the mid-IR emission is optically thin, the mass of the emitting dust with a grain radius $a$ and temperature $T_{d}$ can 
Table 4

SPIRITS 19q Dust Properties with Different Grain Assumptions

\begin{tabular}{|c|c|c|c|c|c|c|}
\hline Grain Composition & $a(\mu \mathrm{m})$ & $T_{\mathrm{d}}(\mathrm{K})$ & $M_{\mathrm{d}}\left(M_{\odot}\right)$ & $\dot{M}_{\mathrm{d}}\left(M_{\odot} \mathrm{yr}^{-1}\right)$ & $\dot{M}_{\mathrm{d}} / \dot{M}_{\mathrm{WR}}^{\mathrm{a}}$ & $\left\langle\dot{M}_{d}\right\rangle^{\mathrm{b}}\left(M_{\odot} \mathrm{yr}^{-1}\right)$ \\
\hline$\left(\rho_{b}=1.8 \mathrm{~g} \mathrm{~cm}^{-3}\right)$ & 0.1 & $690_{-10}^{+10}$ & $6.6_{-0.4}^{+0.4} \times 10^{-6}$ & $6.7_{-0.4}^{+0.4} \times 10^{-6}$ & $30 \%$ & $\lesssim 5.5 \times 10^{-7}$ \\
\hline Graphite & 0.01 & $680_{-10}^{+10}$ & $21.6_{-1.4}^{+1.3} \times 10^{-6}$ & $22.0_{-1.5}^{+1.4} \times 10^{-6}$ & $100 \%$ & $\lesssim 18.0 \times 10^{-7}$ \\
\hline \multirow[t]{2}{*}{$\left(\rho_{b}=2.2 \mathrm{~g} \mathrm{~cm}^{-3}\right)$} & 0.1 & $650_{-10}^{+10}$ & $19.9_{-1.3}^{+1.2} \times 10^{-6}$ & $20.2_{-1.3}^{+1.2} \times 10^{-6}$ & $90 \%$ & $\lesssim 16.6 \times 10^{-7}$ \\
\hline & 0.5 & $760_{-10}^{+10}$ & $2.7_{-0.2}^{+0.2} \times 10^{-6}$ & $2.8_{-0.2}^{+0.2} \times 10^{-6}$ & $10 \%$ & $\lesssim 2.3 \times 10^{-7}$ \\
\hline
\end{tabular}

Notes. Derived SPIRITS $19 \mathrm{q}$ dust temperature $\left(T_{d}\right)$, dust mass $\left(M_{d}\right)$, dust-production rate $\left(\dot{M}_{d}\right)$, dust-to-gas mass fraction $\left(\dot{M}_{\mathrm{d}} / \dot{M}_{\mathrm{WR}}\right)$, and the limit on the periodaveraged dust input rate $\left(\left\langle\dot{M}_{d}\right\rangle\right)$ assuming amorphous carbon or graphite compositions with a grain radius $a=0.01,0.1$, and $0.5 \mu$ m. The amorphous carbon grain emissivity model is adopted from Zubko et al. (1996) and the graphite grain emissivity model is adopted from Draine \& $\mathrm{Li} \mathrm{(2001)} \mathrm{and} \mathrm{Li} \mathrm{\&} \mathrm{Draine} \mathrm{(2001).} \mathrm{The} \mathrm{dust}$ properties are determined from the quiescence-subtracted Spitzer flux on 2018 June 20 (MJD 58289) of $F_{3.6}=1.393 \pm 0.013 \mathrm{mJy}$ and $F_{4.5}=1.655 \pm 0.008 \mathrm{mJy}$. The dust production rate $\dot{M}_{d}$ is derived assuming the dust mass $M_{d}$ is produced over a $\Delta t=0.98 \mathrm{yr}$ timescale.

${ }^{a}$ The mean WC4 mass-loss rate of $2.14 \times 10^{-5} M_{\odot} \mathrm{yr}^{-1}$ derived by Sander et al. (2019) is adopted for $\dot{M}_{\mathrm{WR}}$.

$\mathrm{b}$ The periodicity of the dust formation event in SPIRITS $19 \mathrm{q}$ is assumed to be $\gtrsim 12 \mathrm{yr}$.

be expressed as

$$
M_{d}=\frac{(4 / 3) a \rho_{b} F_{\nu} d^{2}}{Q_{d}(\nu, a) B_{\nu}\left(T_{d}\right)}
$$

where $\rho_{b}$ is the dust grain bulk density, $F_{\nu}$ is the observed flux, $d$ is the distance to SPIRITS $19 \mathrm{q}, Q_{d}(\nu, a)$ is the dust emission efficiency, and $B_{\nu}$ is the Planck function (see Williams et al. 1990). The dust temperature $T_{d}$ can be derived from the ratio of the measured fluxes at 3.6 and $4.5 \mu \mathrm{m}$, where the profile of the IR emission is defined by $F_{\nu} \propto B_{\nu}\left(T_{d}\right) Q_{d}(\nu, a)$. Observations of dust formation from Galactic WC stars suggest that the newly formed dust can have a range of grain sizes from $0.01 \mu \mathrm{m}$ to $\gtrsim 0.5 \mu \mathrm{m}$ (Rajagopal et al. 2007; Williams et al. 2009a; Lau et al. 2020a). The C-rich winds of WC stars and observed featureless mid-IR continuum emission imply that WC dust grains are composed of amorphous carbon and/or graphite (Cherchneff et al. 2000; Zubko et al. 2004; Gupta \& Sahijpal 2020). Given the range of WC dust properties, we estimate the dust properties from SPIRITS $19 \mathrm{q}$ assuming grain emissivity models for amorphous carbon (Zubko et al. 1996) and graphite (Draine \& Li 2001; Li \& Draine 2001) with a uniform grain size radius of $0.01,0.1$, and $0.5 \mu \mathrm{m}$.

The quiescence-subtracted Spitzerflux during the SPIRITS 19q peak IR emission on 2018 June 20 (MJD 58289) was and $F_{3.6}=1.393 \pm 0.013 \mathrm{mJy}$ and $F_{4.5}=1.655 \pm$ $0.008 \mathrm{mJy}$. The observed onset of the rising mid-IR emission on 2017 June 26 (MJD 57930) provides a temporal baseline, $\Delta t$, to estimate the dust production rate from SPIRITS 19q during its IR outburst. The time between the onset of the IR outburst and the IR peak is $\Delta t=0.98 \mathrm{yr}$. We estimate the dust production rates from SPIRITS $19 \mathrm{q}$ with the different grain assumptions based on these flux measurements and summarize the results in Table 4 . The SPIRITS $19 \mathrm{q}$ dust production rates range from $\dot{M}_{d} \approx(2-7) \times 10^{-6} M_{\odot} \mathrm{yr}^{-1}$ for an amorphous carbon composition and $\dot{M}_{d} \approx(3-22) \times 10^{-6} M_{\odot} \mathrm{yr}^{-1}$ for a graphite composition. The SPIRITS 19q dust-production rate during outburst may therefore exceed that of the most efficient Galactic WC dust-makers, WR 112 and WR 104, which exhibit persistent dust production rates of (3-4) $\times 10^{-6} M_{\odot} \mathrm{yr}^{-1}$ (Lau et al. 2020a, 2020b).
It is important to address that SPIRITS 19q exhibits episodic, as opposed to persistent, dust formation. Our observational constraints on the periodicity of dust-formation episodes from SPIRITS $19 q$ provide a lower limit of $\gtrsim 12 \mathrm{yr}$ (Table 3 ). The total period-averaged dust output rate, which is relevant to its contribution to the local dust budget, is therefore a factor of $\gtrsim 12$ lower than the dust production rate measured during the outburst (Table 4). Although the episodic nature of dust formation in SPIRITS 19q reduces the total dust input rate, this does not preclude our observations of enhanced, instantaneous dust formation relative to known Galactic WC dust-makers.

The outflow dust-to-gas mass fraction can be estimated for SPIRITS $19 \mathrm{q}$ by adopting a WC wind mass-loss rate of $\dot{M}_{\mathrm{WR}}=2.14 \times 10^{-5} M_{\odot} \mathrm{yr}^{-1}$, which is consistent with the mean mass-loss rate derived from Galactic WC4 stars by Sander et al. (2019). The derived dust production rates imply dust-to-gas mass fractions of $\sim 10 \%-30 \%$ for amorphous carbon and $\sim 10 \%-100 \%$ for graphite (Table 4 ). Because it is highly unlikely that $90 \%-100 \%$ of the WC wind is forming dust, either the $a=0.1$ and $0.01 \mu \mathrm{m}$ graphite dust models are not valid or the WC star in SPIRITS 19q exhibits a higher mass-loss rate than the mean WC4 mass-loss rate. However, even a $\sim 10 \%$ dust-to-gas mass fraction implies a high dust condensation efficiency given that LMC WC4 stars exhibit carbon surface abundances of $20 \%-45 \%$ (Crowther et al. 2002). Interestingly, this challenges the observational evidence that suggests late-type WC stars (i.e., WC8 and WC9) are the most efficient and heavy dust-forming WC systems (Lau et al. 2020a), which may have major implications on the impact of the dust input from early-type WC stars.

\subsection{Colliding-wind Dust Formation in Early-type WC Binaries}

Dust formation in early-type WC stars, which exhibit hotter temperatures and higher wind velocities than their late-type counterparts, is currently poorly understood given the dearth of known dust-forming WCE systems. Given that these early-type WC stars are likely the dominant population of WC stars in low-metallicity environments, it is important to understand their dust formation and mass-loss properties in order to characterize their impact on the dust abundance and chemistry of their host galaxy's ISM, especially in the early universe. Based on the results of our dust-forming early-type WC candidates and our in-depth analysis of SPIRITS $19 \mathrm{q}$, it is clear 
that the hot photospheres and high wind velocities from WC4 stars do not completely impede the formation of dust.

Due to higher wind velocities, early-type WC stars should exhibit reduced dust formation efficiency relative to late-type WC stars based on the theoretical model of colliding-wind dust formation in WR+OB binaries by Usov (1991). Usov (1991) determined that $\alpha$, the fraction of the WR wind that is compressed and rapidly cooled in the wind-collision shock to form dust, can be characterized as

$$
\alpha \propto \eta^{2}\left(1+\eta^{1 / 2}\right)^{2} \dot{M}_{\mathrm{WR}}^{2} v_{\mathrm{WR}}^{-6} D^{-2},
$$

where $\eta$ is the momentum ratio between the $\mathrm{OB}$ and WR star winds $\left(\eta=\frac{\dot{M}_{\mathrm{OB}} v_{\mathrm{OB}}}{\dot{M}_{\mathrm{WR}} \nu_{\mathrm{WR}}}\right), \dot{M}_{\mathrm{WR} / \mathrm{OB}}$ is the mass-loss rate of the WR/ OB star, $v_{\mathrm{WR} / \mathrm{OB}}$ is the terminal velocity of the WR/OB star wind, and $D$ is the separation distance between the WR and OB stars. In this colliding-wind dust-formation framework, we demonstrate that it is feasible for an early-type WC binary to achieve the same dust-formation efficiency as a late-type WC binary with an identical orbital separation $D$ if the OB companion in the early-type WC binary exhibits a higher mass-loss rate. We note that clumping in the $\mathrm{WC}$ and $\mathrm{OB}$ companion winds (e.g., Smith 2014) may also influence the colliding-wind dust-production efficiency; however, characterizing the effects of clumping is beyond the scope of this work.

Although the average $\dot{M}_{\text {WR }}$ derived for Galactic WC4 stars are consistent with that of Galactic WC9 stars $\left(2.2 \times 10^{-5} M_{\odot}\right.$. $\mathrm{yr}^{-1}$; Sander et al. 2019), the terminal velocity of a WC4 wind $\left(v_{\infty}=3310 \mathrm{~km} \mathrm{~s}^{-1}\right)$ exceeds that of a WC9 wind $\left(v_{\infty}=1390\right.$ $\mathrm{km} \mathrm{s}^{-1}$ ) by more than factor of 2 (Sander et al. 2019). For dustforming $\mathrm{WC} 4+\mathrm{OB}$ and $\mathrm{WC} 9+\mathrm{OB}$ binaries with identical orbital separations, the wind momentum ratio $\eta$ must be enhanced in the $\mathrm{WC} 4+\mathrm{OB}$ system relative to the $\mathrm{WC} 9+\mathrm{OB}$ system by a factor of $\sim 11$ in order to exhibit an equivalent dust-formation efficiency (Equation (5)).

For example, $\eta \sim 0.01$ in the dust-forming WC9+OB binary WR 104 (Soulain et al. 2018), where the OB-companion exhibits a mass-loss rate of $6 \times 10^{-8} M_{\odot} \mathrm{yr}^{-1}$ (Harries et al. 2004). Therefore, a WC4+OB binary with the same $D, v_{\mathrm{OB}}$, and $\dot{M}_{\mathrm{WR}}$ as WR 104 would match its condensation efficiency if the OB-companion possessed a mass-loss rate of $\dot{M}_{\mathrm{OB}} \sim$ $1.6 \times 10^{-6} M_{\odot} \mathrm{yr}^{-1}$. This mass-loss rate is consistent with that of an O supergiant or an early O giant (Muijres et al. 2012). Even at $1 / 3 Z_{\odot}$ metallicity, luminous $\mathrm{O}$ stars are predicted to exhibit mass-loss rates greater than $10^{-6} M_{\odot} \mathrm{yr}^{-1}$ (Vink et al. 2001). The dust-forming WC system HD 36402 in the LMC notably hosts an O8 supergiant companion (Moffat et al. 1990). Not only is it plausible for a WC4 binary to host such a companion, but it is possible for an $\mathrm{O}$ supergiant companion to possess a mass-loss rate greater than $\sim 1.6 \times 10^{-6} M_{\odot} \mathrm{yr}^{-1}$ such that the dust condensation efficiency exceeds that of WR 104. We suggest that this is the reason for the efficient dust production rate measured from SPIRITS 19q (Section 4.1). A companion with a high mass-loss rate may also explain the efficient dust formation from SPIRITS 16df, 14apu, and 18hb inferred from their high absolute [3.6] magnitudes (Figure 8).

It is instructive to address the implications of the limit on the orbital period of SPIRITS $19 \mathrm{q}(P \gtrsim 12 \mathrm{yr})$ on its dust-formation efficiency. This long orbital period implies the semimajor axis of its orbit should be larger than that of WR 104, which exhibits a near-circular orbit with a period of $P \approx 0.66 \mathrm{yr}$ (Tuthill et al.
2008). If SPIRITS $19 \mathrm{q}$ had a circular orbit, this orbital period constraint should lead to a substantially lower dustformation efficiency relative to WR 104 given that $\alpha \propto D^{-2}$ (Equation (5)). However, the sharp IR outburst of SPIRITS 19q (Figure 7) indicates a highly eccentric orbit and thus a timevarying dust-formation efficiency. Dust formation from SPIRITS $19 q$ should therefore peak near periastron passage when the WC star is closest to its companion. We claim that this mechanism is responsible for its IR outburst. Orbital eccentricity is therefore an important parameter that regulates dust formation in long-period WC systems (e.g., Williams et al. 2009a).

Individual WC systems that exhibit high dust formation rates could have a significant impact on the dust input rate in the ISM of galaxies, including those with low metallicities. For example, the dust production rate estimates of SPIRITS 19q during its outburst (Table 4) range between the total dust production rates from the population of RSGs in the LMC $\left(\dot{M}_{d, \mathrm{RSG}}=1.4 \times 10^{-6} M_{\odot} \mathrm{yr}^{-1}\right)$ to the population of AGB stars in the LMC $\left(\dot{M}_{d, \mathrm{AGB}}=1.4 \times 10^{-5} M_{\odot} \mathrm{yr}^{-1}\right.$; Riebel et al. 2012; Srinivasan et al. 2016). Efficient dust production from WC binaries in LMC-like metallicities supports the results from the dust input and binary population synthesis models from Lau et al. (2020a). At LMC-like metallicities with a constant star formation history, Lau et al. (2020a) predicted that WC binaries input dust at a rate comparable with that of AGB stars within factors of a few.

\section{Conclusions}

We have presented Spitzer mid-IR light curves of six extragalactic dust-forming WC candidates in low-metallicity environments from the SPIRITS survey (Figure 7). Over the more than 10-year temporal baselines of Spitzer/IRAC observations at 3.6 and $4.5 \mu \mathrm{m}$, these sources exhibited irregular or episodic mid-IR variability similar to that of known dust-forming WC systems (e.g., Williams 2019). Follow-up optical spectroscopy with Keck/LRIS and HET/ LRS2 of SPIRITS 16ln, 19q, 16df, 14apu, and 18hb confirmed emission from the C IV $\lambda 5801-12$ and/or the C III-IV $\lambda 4650 /$ He II $\lambda 4686$ blend, consistent with early-type WC stars. SPIRITS 14bqe, the only candidate without spectroscopic follow-up, exhibited periodic variability with repeating midIR emission peaks on $\sim 8.5 \mathrm{yr}$ timescales. The periodic mid-IR variability of SPIRITS 14bqe resembles that of the highly eccentric WC5+O9 binary system WR 19 (Figure 10), which shows repeating dust formation and IR-brightening episodes during periastron passage in its $10.1 \mathrm{yr}$ orbit (Williams et al. 2009b).

We identified dust formation from the WC candidates based on their Spitzer [3.6] - [4.5] color. Spitzer/IRAC photometry of all candidates except for SPIRITS 14bqe, which is the most nearby candidate, appeared to suffer from confusion with nearby dusty $\mathrm{H}$ II regions or stellar clusters. After subtracting the quiescent, nonvariable emission, the mid-IR colors from SPIRITS 16ln, 19q, 14apu, and 18hb at their mid-IR peak were consistent with dust formation (Figure 8). During quiescence and outburst, the mid-IR colors of SPIRITS 14bqe were consistent with the presence and formation of dust. Although the irregular mid-IR variability from SPIRITS 16df made it difficult to define and subtract the quiescent mid-IR emission component, its median mid-IR color was consistent with the presence of hot dust. 
We presented SPIRITS $16 \mathrm{ln}$ as the variable mid-IR counterpart of the WC4 + O system N604-WRXc, which also exhibits $\mathrm{X}$-ray variability consistent with a colliding-wind binary (Garofali et al. 2019). This colliding-wind mechanism is associated with dust formation in $\mathrm{WC}+\mathrm{OB}$ binaries, which supports our interpretation of the mid-IR outburst of SPIRIT $16 \mathrm{ln}$ as a dust-formation episode. Based on the spectroscopic classification, X-ray variability, and mid-IR variability, we claimed that SPIRITS $16 \ln / \mathrm{N} 604-\mathrm{WRXc}$ is a dust-forming colliding-wind WC binary. SPIRITS 16ln/N604-WRXc therefore is the first dust-forming colliding-wind WC binary to be identified beyond the LMC.

The absolute [3.6] magnitudes of SPIRITS 19q, 16df, 14apu, and $18 \mathrm{hb}$ exceeded those of one of the brightest known dusty WC systems, WR 104 (Figure 8), which suggests that these candidates may be exhibiting highly efficient dust formation for a WC binary. SPIRITS $19 \mathrm{q}$ exhibited a luminous mid-IR outburst that was significantly brighter than its mid-IR emission in quiescence. We investigated the dust properties from SPIRITS $19 \mathrm{q}$ during outburst using the 3.6 and $4.5 \mu \mathrm{m}$ emission measured from Spitzer. We derived dust-production rates for SPIRITS $19 \mathrm{q}$ ranging from $\dot{M}_{d} \approx(2-7) \times 10^{-6} M_{\odot}$ $\mathrm{yr}^{-1}$ for an amorphous carbon composition and $\dot{M}_{d} \approx$ (3-22) $\times 10^{-6} M_{\odot} \mathrm{yr}^{-1}$ for a graphite composition (Table 4). The SPIRITS $19 \mathrm{q}$ dust-production rate during its outburst may therefore exceed that of the most efficient known dust-forming WC systems $\left(\dot{M}_{d} \approx(3-4) \times 10^{-6} \quad M_{\odot} \quad \mathrm{yr}^{-1}\right.$; Lau et al. 2020a, 2020b).

Based on the theoretical framework of dust production in colliding-wind WR-OB binaries by Usov (1991), we show that efficient dust-formation from early-type WC binaries is plausible with a high mass-loss rate companion such as an $\mathrm{O}$ supergiant or early $\mathrm{O}$ giant star. We therefore suggest that early-type WC systems, which are more common than late-type WC systems in low-metallicity environments, may indeed have a significant impact on the dust input in the ISM of lowmetallicity galaxies. This supports the model results from Lau et al. (2020a), which show that the dust production rate of the population of WC binaries is comparable to that of the AGB star population at LMC-like metallicities with constant star formation histories.

Our results underscore the value of investigating dust formation from early-type WC systems in low-metallicity environments. We also emphasize the importance of the continued exploration of dust production from WC systems within our Galaxy, where spatially resolved observations allow us to study the interplay between dust formation and the orbital and mass-loss properties of the central WC binary (e.g., Callingham et al. 2019; Han et al. 2020; Lau et al. 2020b). Spatially resolved observations of dust formed by WC binaries are also crucial for probing how the newly formed dust evolves as it expands and integrates into the surrounding ISM (e.g., Marchenko et al. 2002; Williams et al. 2009a). Last, our results demonstrate the necessity of ongoing long-term IR photometric monitoring programs such as the recently commissioned Palomar Gattini-IR (De et al. 2020) for detecting episodic variations that would otherwise be missed.

We acknowledge S. Anand and C. Fremling for their support on the observations obtained with Keck I/LRIS. We thank N. Morrell, P. Mudumba, and R. Gehrz for their valuable feedback on our study. We also thank the anonymous referee for their insightful review and comments that have improved the quality of our work. R.M.L. acknowledges the Japan Aerospace Exploration Agency's International Top Young Fellowship (ITYF). A.F.J.M. is grateful for financial assistance from NSERC (Canada). This work is based on observations and archival data obtained with the Spitzer Space Telescope, which was operated by the Jet Propulsion Laboratory, California Institute of Technology under a contract with NASA. Support for this work was provided by NASA through an award issued by JPL/Caltech. Some of the data presented herein were obtained at the W. M. Keck Observatory, which is operated as a scientific partnership among the California Institute of Technology, the University of California and the National Aeronautics and Space Administration. The Observatory was made possible by the generous financial support of the W. M. Keck Foundation. The authors wish to recognize and acknowledge the very significant cultural role and reverence that the summit of Maunakea has always had within the indigenous Hawaiian community. We are most fortunate to have the opportunity to conduct observations from this mountain. The Hobby-Eberly Telescope (HET) is a joint project of the University of Texas at Austin, the Pennsylvania State University, Ludwig-Maximilians-Universität München, and Georg-August-Universität Göttingen. The HET is named in honor of its principal benefactors, William P. Hobby and Robert E. Eberly. The Low Resolution Spectrograph 2 (LRS2) was developed and funded by the University of Texas at Austin McDonald Observatory and Department of Astronomy and by Pennsylvania State University. We thank the Leibniz-Institut für Astrophysik Potsdam (AIP) and the Institut für Astrophysik Göttingen (IAG) for their contributions to the construction of the integral field units. Based partially on observations made with the NASA/ESA Hubble Space Telescope, obtained from the data archive at the Space Telescope Science Institute. STScI is operated by the Association of Universities for Research in Astronomy, Inc. under NASA contract NAS 5-26555.

Facilities: Spitzer(IRAC), Keck:I (LRIS), Keck:II (NIRES), HET(LRS2), P200(WIRC), HST(ACS/WFC)

Software: Astropy (Astropy Collaboration et al. 2013, 2018), LPipe (Perley 2019), Spex-tool (Cushing et al. 2004), Panacea (https://github.com/grzeimann/Panacea).

\section{ORCID iDs}

Matthew J. Hankins (1) https://orcid.org/0000-0001-9315-8437 Mansi M. Kasliwal iㅏ https://orcid.org/0000-0002-5619-4938 Howard E. Bond (i) https://orcid.org/0000-0003-1377-7145 Nathan Smith (1) https://orcid.org/0000-0001-5510-2424 Peredur M. Williams (i) https://orcid.org/0000-00028092-980X

\section{References}

Allen, D. A., Swings, J. P., \& Harvey, P. M. 1972, A\&A, 20, 333 Anand, G. S., Rizzi, L., \& Tully, R. B. 2018, AJ, 156, 105

Asplund, M., Grevesse, N., Sauval, A. J., et al. 2009, ARA\&A, 47, 481

Astropy Collaboration, Price-Whelan, A. M., Sipőcz, B. M., et al. 2018, AJ, 156,123

Astropy Collaboration, Robitaille, T. P., Tollerud, E. J., et al. 2013, A\&A, 558, A33

Bartzakos, P., Moffat, A. F. J., \& Niemela, V. S. 2001, MNRAS, 324, 18 Bonanos, A. Z., Massa, D. L., Sewilo, M., et al. 2009, AJ, 138, 1003 Bond, H. E., Bedin, L. R., Bonanos, A. Z., et al. 2009, ApJL, 695, L154 Bresolin, F., Urbaneja, M. A., Gieren, W., et al. 2007, ApJ, 671, 2028 Callingham, J. R., Tuthill, P. G., Pope, B. J. S., et al. 2019, NatAs, 3, 82 
Cherchneff, I., Le Teuff, Y. H., Williams, P. M., et al. 2000, A\&A, 357, 572 Chonis, T. S., Hill, G. J., Lee, H., et al. 2016, Proc. SPIE, 9908, 99084C Crowther, P. A. 2007, ARA\&A, 45, 177

Crowther, P. A., De Marco, O., \& Barlow, M. J. 1998, MNRAS, 296, 367

Crowther, P. A., Dessart, L., Hillier, D. J., et al. 2002, A\&A, 392, 653

Croxall, K. V., Pogge, R. W., Berg, D. A., et al. 2016, ApJ, 830, 4

Cushing, M. C., Vacca, W. D., \& Rayner, J. T. 2004, PASP, 116, 362

De, K., Hankins, M. J., Kasliwal, M. M., et al. 2020, PASP, 132, 025001

Draine, B. T., \& Li, A. 2001, ApJ, 551, 807

Drissen, L., Roy, J.-R., Moffat, A. F. J., et al. 1999, AJ, 117, 1249

Dsilva, K., Shenar, T., Sana, H., et al. 2020, A\&A, 641, A26

Eldridge, J. J., Stanway, E. R., Xiao, L., et al. 2017, PASA, 34, e058

Fazio, G. G., Hora, J. L., Allen, L. E., et al. 2004, ApJS, 154, 10

Garnett, D. R., Shields, G. A., Skillman, E. D., et al. 1997, ApJ, 489, 63

Garofali, K., Levesque, E. M., Massey, P., et al. 2019, ApJ, 880, 8

Gehrz, R. D., \& Hackwell, J. A. 1974, ApJ, 194, 619

Gehrz, R. D., Roellig, T. L., Werner, M. W., et al. 2007, RScI, 78, 011302

Gieren, W., Górski, M., Pietrzyński, G., et al. 2013, ApJ, 773, 69

Goodrich, R., \& Cohen, M. 2003, Proc. SPIE, 4843, 146

Groh, J. H., Damineli, A., \& Jablonski, F. 2007, A\&A, 465, 993

Gupta, A., \& Sahijpal, S. 2020, MNRAS, 492, 2058

Hackwell, J. A., Gehrz, R. D., \& Grasdalen, G. L. 1979, ApJ, 234, 133

Hadfield, L. J., \& Crowther, P. A. 2007, MNRAS, 381, 418

Han, Y., Tuthill, P. G., Lau, R. M., et al. 2020, MNRAS, 498, 5604

Harries, T. J., Monnier, J. D., Symington, N. H., et al. 2004, MNRAS, 350, 565

Hatt, D., Beaton, R. L., Freedman, W. L., et al. 2017, ApJ, 845, 146

Howarth, I. D., \& Schmutz, W. 1992, A\&A, 261, 503

Jencson, J. E., Kasliwal, M. M., Adams, S. M., et al. 2019, ApJ, 886, 40

Karambelkar, V. R., Adams, S. M., Whitelock, P. A., et al. 2019, ApJ, 877, 110

Kasliwal, M. M., Bally, J., Masci, F., et al. 2017, ApJ, 839, 88

Khramtsova, M. S., Wiebe, D. S., Lozinskaya, T. A., et al. 2014, MNRAS, 444,757

Lau, R. M., Eldridge, J. J., Hankins, M. J., et al. 2020a, ApJ, 898, 74

Lau, R. M., Hankins, M. J., Han, Y., et al. 2020b, ApJ, 900, 190

Lau, R. M., Heida, M., Walton, D. J., et al. 2019, ApJ, 878, 71

Li, A., \& Draine, B. T. 2001, ApJ, 554, 778

Magrini, L., Vílchez, J. M., Mampaso, A., et al. 2007, A\&A, 470, 865

Mainzer, A., Bauer, J., Cutri, R. M., et al. 2014, ApJ, 792, 30

Marchenko, S. V., Moffat, A. F. J., Vacca, W. D., et al. 2002, ApJL, 565, L59

Martínez-González, S., Wünsch, R., Silich, S., et al. 2019, ApJ, 887, 198

Massey, P., \& Johnson, O. 1998, ApJ, 505, 793

Meixner, M., Gordon, K. D., Indebetouw, R., et al. 2006, AJ, 132, 2268

Melena, N. W., Elmegreen, B. G., Hunter, D. A., et al. 2009, AJ, 138, 1203

Moffat, A. F. J., Niemela, V. S., \& Marraco, H. G. 1990, ApJ, 348, 232

Morris, P. W., Brownsberger, K. R., Conti, P. S., et al. 1993, ApJ, 412, 324

Moustakas, J., Kennicutt, R. C., Tremonti, C. A., et al. 2010, ApJS, 190, 233

Muijres, L. E., Vink, J. S., de Koter, A., et al. 2012, A\&A, 537, A37
Neugent, K. F., Massey, P., \& Morrell, N. 2018, ApJ, 863, 181

Perley, D. A. 2019, PASP, 131, 084503

Pilyugin, L. S., \& Thuan, T. X. 2005, ApJ, 631, 231

Radburn-Smith, D. J., de Jong, R. S., Seth, A. C., et al. 2011, ApJS, 195, 18

Rajagopal, J., Menut, J.-L., Wallace, D., et al. 2007, ApJ, 671, 2017

Rate, G., \& Crowther, P. A. 2020, MNRAS, 493, 1512

Rayner, J. T., Cushing, M. C., \& Vacca, W. D. 2009, ApJS, 185, 289

Riebel, D., Srinivasan, S., Sargent, B., et al. 2012, ApJ, 753, 71

Rosslowe, C. K., \& Crowther, P. A. 2015, MNRAS, 447, 2322

Sander, A. A. C., Hamann, W.-R., Todt, H., et al. 2019, A\&A, 621, A92

Shappee, B. J., \& Stanek, K. Z. 2011, ApJ, 733, 124

Smith, N. 2014, ARA\&A, 52, 487

Smith, N., Andrews, J. E., Van Dyk, S. D., et al. 2016, MNRAS, 458, 950

Soulain, A., Millour, F., Lopez, B., et al. 2018, A\&A, 618, A108

Srinivasan, S., Boyer, M. L., Kemper, F., et al. 2016, MNRAS, 457, 2814

Stevens, I. R., Blondin, J. M., \& Pollock, A. M. T. 1992, ApJ, 386, 265

Temim, T., Dwek, E., Tchernyshyov, K., et al. 2015, ApJ, 799, 158

Torres, A. V., \& Massey, P. 1987, ApJS, 65, 459

Tuthill, P. G., Monnier, J. D., \& Danchi, W. C. 1999, Natur, 398, 487

Tuthill, P. G., Monnier, J. D., Lawrance, N., et al. 2008, ApJ, 675, 698

Usov, V. V. 1991, MNRAS, 252, 49

Vacca, W. D., Cushing, M. C., \& Rayner, J. T. 2003, PASP, 115, 389

van der Hucht, K. A., Morris, P. W., Williams, P. M., et al. 1996, A\&A, 315, L193

Veen, P. M., van der Hucht, K. A., Williams, P. M., et al. 1998, A\&A, 339, L45

Véron, P., \& Sauvayre, A. 1965, AnAp, 28, 698

Vilchez, J. M., Pagel, B. E. J., Diaz, A. I., et al. 1988, MNRAS, 235, 633

Vink, J. S., de Koter, A., \& Lamers, H. J. G. L. M. 2001, A\&A, 369, 574

Werner, M. W., Roellig, T. L., Low, F. J., et al. 2004, ApJS, 154, 1

Williams, P. M. 2019, MNRAS, 488, 1282

Williams, P. M., Beattie, D. H., Lee, T. J., et al. 1978, MNRAS, 185, 467

Williams, P. M., Chu, Y.-H., Gruendl, R. A., et al. 2013, MNRAS, 431, 1160

Williams, P. M., Marchenko, S. V., Marston, A. P., et al. 2009a, MNRAS, 395, 1749

Williams, P. M., Rauw, G., \& van der Hucht, K. A. 2009b, MNRAS, 395, 2221

Williams, P. M., van der Hucht, K. A., Pollock, A. M. T., et al. 1990, MNRAS, 243, 662

Williams, P. M., van der Hucht, K. A., \& Thé, P. S. 1987, A\&A, 182, 91

Williams, P. M., van der Hucht, K. A., The, P. S., et al. 1990, MNRAS, $247,18 \mathrm{P}$

Wilson, J. C., Eikenberry, S. S., Henderson, C. P., et al. 2003, Proc. SPIE, 4841,451

Yukita, M., Swartz, D. A., Tennant, A. F., et al. 2010, AJ, 139, 1066

Zubko, V., Dwek, E., \& Arendt, R. G. 2004, ApJS, 152, 211

Zubko, V. G. 1998, MNRAS, 295, 109

Zubko, V. G., Mennella, V., Colangeli, L., et al. 1996, MNRAS, 282, 1321 